\title{
Cosmology dependence of galaxy cluster scaling relations
}

\author{
Priyanka Singh ${ }^{1,2} \star$, Alex Saro ${ }^{1,2,3}$, Matteo Costanzi ${ }^{1,2}$, Klaus Dolag $^{4,5}$ \\ 1 INAF-Osservatorio Astronomico di Trieste, via G. B. Tiepolo 11, I-34143 Trieste, Italy \\ 2 IFPU - Institute for Fundamental Physics of the Universe, Via Beirut 2, 34014 Trieste, Italy \\ 3 Astronomy Unit, Department of Physics, University of Trieste, via Tiepolo 11, I-34131 Trieste, Italy \\ 4 University Observatory Munich, Scheinerstr 1, D-81679 Munich, Germany \\ 5 Max-Planck-Institut für Astrophysik (MPA), Karl-Schwarzschild Strasse 1, D-85748 Garching bei München, Germany
}

14 April 2020

\begin{abstract}
The abundance of galaxy clusters as a function of mass and redshift is a well known powerful cosmological probe, which relies on underlying modelling assumptions on the mass-observable relations (MOR). Some of the MOR parameters can be constrained directly from multi-wavelength observations, as the normalization at some reference cosmology, the mass-slope, the redshift evolution and the intrinsic scatter. However, the cosmology dependence of MORs cannot be tested with multi-wavelength observations alone. We use Magneticum simulations to explore the cosmology dependence of galaxy cluster scaling relations. We run fifteen hydro-dynamical cosmological simulations varying $\Omega_{m}, \Omega_{b}, h_{0}$ and $\sigma_{8}$ (around a reference cosmological model). The MORs considered are gas mass, baryonic mass, gas temperature, $Y$ and velocity dispersion as a function of virial mass. We verify that the mass and redshift slopes and the intrinsic scatter of the MORs are nearly independent of cosmology with variations significantly smaller than current observational uncertainties. We show that the gas mass and baryonic mass sensitively depends only on the baryon fraction, velocity dispersion and gas temperature on $h_{0}$, and $Y$ on both baryon fraction and $h_{0}$. We investigate the cosmological implications of our MOR parameterization on a mock catalog created for an idealized eROSITA-like experiment. We show that our parametrization introduces a strong degeneracy between the cosmological parameters and the normalization of the MOR. Finally, the parameter constraints derived at different overdensity $\left(\Delta_{500 c}\right)$, for X-ray bolometric gas luminosity, and for different subgrid physics prescriptions are shown in the appendix.
\end{abstract}

Key words: cosmology: large-scale structure of Universe

\section{Introduction}

Galaxy clusters are the most massive gravitationally bound structures in the Universe and represent a well known powerful cosmological tool. Their abundance as a function of redshift and mass is sensitive to both the expansion history and the history of structure formation in the Universe (see Allen, Evrard \& Mantz 2011 for a review), providing therefore complementary information to purely geometric probes such as Type Ia supernovae, the primary cosmic microwave background (CMB) and baryonic acoustic oscillations.

Integrated observable properties of galaxy clusters like $\mathrm{X}$-ray luminosity and temperature, the optical richness and their associated velocity dispersion, and the intensity of the

\footnotetext{
* priyanka.singh@inaf.it
}

Sunyaev-Zeldovich effect (SZE: Sunyaev \& Zel'dovich 1972), are generally used as a proxy for the total cluster mass, as they are expected to regularly scale with galaxy cluster mass following mass-observable scaling relations (MOR), although with some associated intrinsic scatter. Current studies of the cluster mass function (often described as cluster number-counts experiments) are therefore simultaneously exploring both cosmological and MOR (including the intrinsic scatter) parameters to constrain cosmological models (e.g., Planck Collaboration et al. 2016. Mantz et al. 2015. Bocquet et al. 2019). The standard approach for these stateof-the-art studies is to calibrate the MOR empirically, by anchoring the associated parameters through either weaklensing (e.g. Bardeau et al. 2007, Okabe et al. 2010, Hoekstra et al. 2012 | Applegate et al. 2014, Hoekstra et al. 2015. Hilton et al. 2018 (Dietrich et al. 2019) or through dynam- 
ical studies (e.g. Sifón et al. 2013 Hasselfield et al. 2013 Bocquet et al. 2015 Capasso et al. 2019a b c), methods which are more directly linked to effect of gravity alone, and thus easy to characterize in terms of systematics associated with the treatment of the complex physics regulating the baryonic component. Therefore, for this reason, biases associated with weak lensing and dynamical estimates can be calibrated more robustly with numerical simulations. As a result, MOR parameters including the normalization, the mass slope, the redshift evolution, and the scatter, can be directly constrained from multi-wavelength observations (Mantz et al. 2015; Dietrich et al. 2019 Bocquet et al. 2019). In most of these studies, the cosmological dependence of MORs is usually assumed to be only related to the background evolution of the Universe, with the notable exception of measurements of the baryon fraction in galaxy cluster to constraint the matter density (e.g., Mantz et al. 2014).

Within this framework, numerical and hydro-dynamical cosmological simulations still provide fundamental information:

(i) they provide accurate calibration of the theoretical halo mass function (e.g., Tinker et al. 2008, Cui, Borgani \& Murante 2014, Velliscig et al.|2014 Bocquet et al.|2015. Despali et al. 2016, McClintock et al. 2019, and references therein);

(ii) they provide accurate calibration of possible biases affecting the observables used to anchor the absolute scale of the MOR, as weak-lensing and dynamical mass calibration (e.g., White, Cohn \& Smit 2010, Saro et al. 2013, Becker \& Kravtsov 2011 | Rasia et al. 2012, and references therein);

(iii) they provide guidance on the functional form for the mean relation and associated intrinsic scatter of the MOR, as well as priors on parameters that observationally are on weakly constraint (Stanek et al. 2009, Truong et al. 2018 Gupta et al. 2016).

In particular, as we have access to only one observable Universe, these simulations represent the only way to test if the MORs are cosmology dependent and, if they are, to calibrate the parameters describing this dependence.

In this paper, we explore the cosmology dependence of galaxy cluster scaling relations using cluster catalogs identified in the suite of Magneticum simulations Th $^{-}$These large cosmological simulations are designed to investigate different cosmological scales with very large number of particles and, at the same time, to describe the hydro-dynamical evolution of the baryonic collisional component. They therefore provide a complementary tool with respect to purely $\mathrm{N}$ body simulations such as Quijote simulations (VillaescusaNavarro et al. 2019), the Mira-Titan Universe (Heitmann et al.|2016, Lawrence et al. 2017) and Aemulus simulations (DeRose et al. 2019). Furthermore, due to the large simulated cosmological volumes, they are better designed to study the cluster population with respect to other higherresolution, but smaller volumes simulations such as, e.g., the BAHAMAS simulations (McCarthy et al. 2018. Stafford et al. 2019). Moreover, the purpose of Magneticum simulations is to provide a theoretical counterpart for Large Scale Structure (LSS), therefore the dynamical range of the cosmological parameters space explored is significantly broader than

† www.magneticum.org the current CMB cosmological constraints (Planck Collaboration et al. 2017), currently tested in other studies (e.g. Aemulus, BAHAMAS simulations).

In this work, we run Magneticum simulation for fifteen different cosmological volumes, each one generated with the same initial seeds, but with different cosmological parameters. All the simulations include the description of the same physical processes and use the same sub-grid model parameters. Our basic assumption is that the variation of the cosmological model should not directly affect the microscopic processes that these sub-grid parameters describe and therefore, these sub-grid physics parameters have been tuned to reproduce observed properties of galaxy clusters at an arbitrarily (but reasonable, and consistent with observations) chosen reference cosmology (Bocquet et al. 2016, Gupta et al. 2016, Dolag, Mevius \& Remus |2017, Remus, Dolag \& Hoffmann 2017 | Biffi, Dolag \& Merloni|2018, Ragagnin et al.|2019). In other words, their numerical value does not carry any physical meaning. As a result, the results presented in this work could be considered robust only if they are independent of the (arbitrary) choice of the reference cosmology used to tune the sub-grid model parameters. Instead of retuning them for each different cosmological model (an effort which would require an unfeasible computational cost), we address the robustness of our results by studying the validity of our model assumption in the adopted functional form of the MORs. In particular, we explicitly verify that the cosmological dependence of the MORs is only affecting the normalization of the studied scaling relations, but does not change the mass slope, the redshift evolution, and the intrinsic scatter. Therefore, we argue that re-tuning the subgrid model parameters for different choices of the reference cosmology would not impact the cosmological dependence of the MOR, as it will only translate into a different zero-point normalization.

In summary, the aim of this paper is: $i$ ) to construct a universal scaling relation where the cosmology dependence of the scaling relation is absorbed in its amplitude, $i i$ ) to test the robustness of our parameterization with respect to the observational uncertainties, and $i i i$ ) to forecast the impact our cosmology dependent parameterization on an idealized cluster cosmology experiment. This paper is organized as follows: in Section 2 we briefly describe the details of the simulation setup. In Section 3 we describe the basic ingredients of the MOR and test its robustness. In Section 4 , we present the results of our analysis for $M_{\text {gas }}, T_{\text {gas }}, Y$ and $\sigma_{v}$. In Section 5 , we discuss the impact of our MOR parametrization for an idealized cosmology experiment. In Section 6, we present the summary of the main analysis.

\section{Simulation details}

Magneticum simulations are based on the Smoothed Particle Hydrodynamics (SPH) code P-GADGET3 which itself is an improved version of P-GADGET2 (Springel 2005, Springel et al. 2005). The simulation includes a variety of physical processes such as metallicity dependent radiative cooling (Wiersma, Schaye \& Smith 2009), UV background heating (Haardt \& Madau 2001), a detailed model of star formation, chemical enrichment (Tornatore et al. 2007) and supernovae $(\mathrm{SNe})$ as well as active galactic nuclei (AGN) driven feed- 
Cosmological dependence in MOR

Table 1. Cosmological parameter values for the fifteen simulation boxes.

\begin{tabular}{c|ccccccccccccccc} 
& $\mathrm{C} 1$ & $\mathrm{C} 2$ & $\mathrm{C} 3$ & $\mathrm{C} 4$ & $\mathrm{C} 5$ & $\mathrm{C} 6$ & $\mathrm{C} 7$ & $\mathrm{C} 8$ & $\mathrm{C} 9$ & $\mathrm{C} 10$ & $\mathrm{C} 11$ & $\mathrm{C} 12$ & $\mathrm{C} 13$ & $\mathrm{C} 14$ & $\mathrm{C} 15$ \\
\hline$\Omega_{m}$ & 0.153 & 0.189 & 0.200 & 0.204 & 0.222 & 0.232 & 0.268 & 0.272 & 0.301 & 0.304 & 0.342 & 0.363 & 0.400 & 0.406 & 0.428 \\
$\Omega_{b}$ & 0.0408 & 0.0455 & 0.0415 & 0.0437 & 0.0421 & 0.413 & 0.0449 & 0.0456 & 0.0460 & 0.0504 & 0.0462 & 0.0490 & 0.0485 & 0.0466 & 0.0492 \\
$\sigma_{8}$ & 0.614 & 0.697 & 0.850 & 0.739 & 0.793 & 0.687 & 0.721 & 0.809 & 0.824 & 0.886 & 0.834 & 0.884 & 0.650 & 0.867 & 0.830 \\
$h_{0}$ & 0.666 & 0.703 & 0.730 & 0.689 & 0.676 & 0.670 & 0.699 & 0.704 & 0.707 & 0.740 & 0.708 & 0.729 & 0.675 & 0.712 & 0.732 \\
$f_{b}$ & 0.267 & 0.241 & 0.208 & 0.214 & 0.190 & 0.178 & 0.168 & 0.168 & 0.153 & 0.166 & 0.135 & 0.135 & 0.121 & 0.115 & 0.115
\end{tabular}

back prescriptions (Springel \& Hernquist 2003, Di Matteo et al. 2008, Fabian |2010| Hirschmann et al. |2014; | Bocquet et al. 2016).

For the purpose of our study, we use Box1a from Magneticum simulation set, which is a large size, medium resolution box. The size of the box is $\sim 896 h_{0}^{-1} \mathrm{Mpc}$. It contains $1526^{3}$ dark matter particles and an equal number of gas particles. For our reference cosmology, this corresponds to a characteristic mass resolution of dark matter, gas and star particles of $1.3 \times 10^{10} h_{0}^{-1} M_{\odot}, 2.6 \times 10^{9} h_{0}^{-1} M_{\odot}$ and $6.5 \times 10^{8} h_{0}^{-1} M_{\odot}$, respectively. The gravitational softening lengths for dark matter, gas and star particles are $10 h_{0}^{-1} \mathrm{kpc}$, $10 h_{0}^{-1} \mathrm{kpc}$ and $5 h_{0}^{-1} \mathrm{kpc}$, respectively.

We run the same simulation set-up for a sample of fifteen different flat $\Lambda \mathrm{CDM}$ cosmological models $(\mathrm{C} 1, \mathrm{C} 2, \ldots$, $\mathrm{C} 15)$. The cosmological parameters varied in each simulations are $\Omega_{m}, \sigma_{8}, h_{0}$, and $\Omega_{b}$ and their values are specified in Table 1 and shown in Figure 1 as coloured points, together with cosmological constraints obtained by state-ofthe-art cluster number counts experiment (Bocquet et al. 2019 , with an additional Gaussian prior on $h_{0}$ with mean 0.704 and width 0.014 ). The parameter ranges used are thus $0.15<\Omega_{m}<0.45,0.6<\sigma_{8}<0.9$, and $0.65<h_{0}<0.75$, to cover the entire dynamic range of current large-scalestructure cosmological constraints. The cosmological parameters are chosen from above ranges using Latin hypercube sampling $f$ The cosmologies are labelled as $\mathrm{C} 1, \mathrm{C} 2, \ldots ., \mathrm{C} 15$ in order of increasing value of $\Omega_{m}$. Note that, thirteen out of fifteen cosmologies have fixed $\Omega_{b} h_{0}^{-2} \sim 0.092$, perpendicular to the direction of degeneracy between $\Omega_{b}$ and $h_{0}$. The cosmologies $\mathrm{C} 3$ and $\mathrm{C} 13$ have been added to break the degeneracy between the two. Our reference cosmology (C8) corresponds to WMAP7 best fit results (Komatsu et al. 2011).

Friends-of-friends (FoF) algorithm is used to identify haloes, linking only the dark matter particles with a linking length $\mathrm{b}=0.16$. A SUBFIND algorithm (Springel et al. 2001; Dolag et al. 2009) is used to compute spherical overdensity (SO) virial mass (Mvir, Bryan \& Norman 1998), where, $\Delta_{\text {vir }}$ is a function of $C_{i}$. The halo is centred at the position of the dark matter particle in a FoF group having the minimum value of the gravitational potential. The observable quantities are integrated within the virial radius for each of the identified halo. Specifically, we study the gas mass $\left(M_{\text {gas }}\right)$, the gas temperature $\left(T_{\text {gas }}\right)$, the X-ray pseudo-

\footnotetext{
$\ddagger$ https://pythonhosted.org/pyDOE/randomized.html
}

pressure $Y\left(\equiv M_{\text {gas }} \times T_{\text {gas }}\right)$, where $M_{\text {gas }}$ is the sum of mass of all gas particles within a given overdensity radius, and $T_{\text {gas }}$ is the associated mass weighted gas temperature. Furthermore we also investigate the mass- $\sigma_{v}$ relation, where the velocity dispersion $\sigma_{v}$ is the mass weighted velocity dispersion of all the particles belonging to each main halo. Results for X-ray bolometric gas luminosity and stellar mass are given in Appendix $\mathrm{A}$ and 4.3 respectively. Results for $M_{500 c}\left(\Delta_{500 c}=500\right.$ w.r.t. $\left.\rho_{c}\right)$ are given in Appendix B

We apply conservative mass cuts and select only haloes with $M_{\text {vir }}>2 \times 10^{14} M_{\odot}$ (corresponding to approximately $10^{4}$ particles), to ensure that haloes extracted from the hydro simulations are not affected by issues related to resolution and numerical artifacts. We extract cluster catalogues at six redshifts. The final number of haloes used in each simulation and redshift is shown in Table 2

We note that all the above mentioned observables are representing simplified and idealized versions of the actual physical observed quantities. A large effort has been dedicated over the last decades (e.g., Nagai, Kravtsov \& Vikhlinin 2007, Avestruz et al. 2014) to understand how the physical properties of haloes translate into observables (and vice-versa) through the analysis of dedicated mocks. These mock simulation works quantifies observational biases and scatter in observationally derived quantities, such as gas mass (Nagai \& Lau 2011, Zhuravleva et al. 2013), Xray temperature, $Y$ (Khedekar et al. 2013|| Rasia et al. 2014), and velocity dispersion (Lau, Nagai \& Kravtsov 2010 Saro et al. 2013 Munari et al. 2013), and hydrostatic mass (Lau, Kravtsov \& Nagai 2009, Lau, Nagai \& Nelson 2013, Nelson, Lau \& Nagai |2014 | Biffi et al.|2016; Shi et al.||2016). This project is however focused only on the cosmological dependence of the MORs, which we thus assume to be unrelated to the biases and scatter associated to such observational effects.

\section{Method}

For a given observable $O$, our aim is to construct a universal scaling relation, described by a set of parameters which are therefore assumed to be all mass, redshift and cosmology independent. We adopt the following functional form to describe the MOR: 


$$
\begin{array}{r}
\ln O=\Pi_{c}+\left(\alpha+\alpha_{s s}\right) \ln \left(\frac{M}{M_{P}}\right)+\beta_{s s} \ln \left(\frac{F(z)}{F\left(z_{P}\right)}\right) \\
+\beta \ln \left(\frac{1+z}{1+z_{P}}\right) \pm \sigma
\end{array}
$$

where $F(z) \equiv E(z) \sqrt{\Delta_{\text {vir }}(z)}, \sigma$ is the intrinsic log-normal scatter, $\alpha_{s s}$ and $\beta_{s s}$ (see Table 3 represent the self-similar mass and redshift dependence of the MOR (Böhringer, Dolag \& Chon 2012), and $M_{P}=2.85 \times 10^{14} M_{\odot}$ and $z_{P}=0.14$ are the pivot mass and redshift (the median mass and redshift of the sample). The parameters $\alpha$ and $\beta$ capture any deviations from the predicted self-similar mass dependence and evolution, and are therefore both zero in a perfectly self-similar scenario. We choose a simple $1+\mathrm{z}$ parameterization to capture the redshift evolution of the MORs, separating it from the traditional $E(z)$ evolution. This way, we remove the expected cosmology dependent part (the $\mathrm{E}(\mathrm{z})$ term) associated to the self-similar evolution and be agnostic on the remaining deviations. The term $\Pi_{c}$ is the normalization of the MOR containing its cosmological dependencies. We assume a simple power law dependence of normalization on cosmological parameters.

$$
\Pi_{c}=\Pi_{c, 0}+\gamma_{h_{0}} \ln \left(\frac{h_{0}}{h_{0}^{P}}\right)+\gamma_{b} \ln \left(\frac{f_{b}}{f_{b}^{P}}\right)+\gamma_{\sigma_{8}} \ln \left(\frac{\sigma_{8}}{\sigma_{8}^{P}}\right)
$$

where $f_{b}=\Omega_{b} / \Omega_{m}$ is the cosmic baryon fraction, and $h_{0}^{P}=$ $0.704, f_{b}^{P}=0.168$ and $\sigma_{8}^{P}=0.809$ are the pivot points in cosmological parameters equal to our C8 cosmology. Note that, the cosmology dependence of $\Pi_{c}$ should be viewed as an "effective cosmology dependence" when the commonly used functional form of scaling relations is adopted.

The adopted MOR functional form (Equation 1 and 2 consists therefore in two distinct families of parameters:

- Astrophysical parameters: $\Pi_{c, 0}, \alpha, \beta$ and $\sigma$. These are the parameters that can be observationally constrained using multi-wavelength data.

- Cosmological parameters $\gamma_{\mathrm{s}:} \gamma_{h_{0}}, \gamma_{b}$ and $\gamma_{\sigma_{8}}$. These are the parameters that cannot be constrained with observations.

The primary purpose of this work is to provide the best estimates and most reliable uncertainties associated to the parameters describing the cosmology dependence of the MOR $\gamma \mathrm{s}$. Note that, a running of $\Pi_{c}$ (i.e. a non-zero value of $\gamma$ ) does not imply a causation relation between the cosmological parameters and the scaling relation parameters. It incorporates any possible correlation between the MORs and the derived cosmology dependent quantities.

\subsection{Robustness of the model}

The main assumption that goes into constructing Equation 1 and 2 is that $\alpha, \beta$ and $\sigma$ are cosmology independent, or, in other words, that cosmology is only affecting the normalization of the MOR. To test that our assumption is indeed justified, we fit the MOR (after applying a $3 \sigma$ clipping to remove outliers) separately on each individual cosmological simulation. More in detail, for each $\mathrm{C} i$ simulation we use a Gaussian likelihood and uninformative priors (with a support significantly broader than the recovered posterior probability distribution) and fit only for $\Pi_{c, 0}, \alpha, \beta$ and $\sigma$ using Equation 1

$$
\begin{array}{r}
\ln \mathcal{L}=-\frac{1}{2} \sum_{j}\left[\left(\frac{\ln O\left(\Pi_{c, 0}, \alpha, \beta, \sigma, M_{j}, z_{j}\right)-\ln O_{j}}{\sigma}\right)^{2}\right. \\
\left.+\ln \left(2 \pi \sigma^{2}\right)\right]
\end{array}
$$

where $M_{j}$ and $z_{j}$ are respectively the mass and redshift of $j^{t h}$ halo. The parameter space is explored with the emcee affineinvariant sampler (Foreman-Mackey et al. 2013) to find the best-fitting values and associated uncertainties.

Coloured points in Figures 2 and 3 show the variation of $\alpha, \beta$ and $\sigma$ for the 15 different simulated cosmologies. The overall resulting range is highlighted by the darker grey bands. While residual trends and variations in these parameters are clearly visible (e.g., decreasing $\alpha$ for $M_{\text {gas }}$ from C1 to C15), we note that these differences are much smaller than current state-of-the-art observational constraints (lighter grey bands enclosed by dashed lines in Figures 2 and 3 for the three X-ray observables Bulbul et al. (2019) (hereafter, B19) and by previous studies on simulations for the $\sigma_{v}-M$ relation (Evrard et al. 2008). This result therefore justifies our assumption about the cosmology independence of $\alpha, \beta$ and $\sigma$ in Equation 1. In other words, any cosmology dependence introduced in these parameters due to the presence of baryons is only of second order. Note that, the cosmology dependence of these astrophysical parameters cannot be neglected once it becomes comparable to the observational uncertainties. As next generation surveys will approach this limit, these approximations will be no longer acceptable and a much more careful description of the cosmological dependence of the MOR will be required, including wider parametrizations or relying on different approaches (e.g., emulators Quijote, Mira-Titan Universe, Aemulus).

In order to estimate how the residual lack of universality on the astrophysical parameters propagates into systematic variations on the cosmological parameters $\gamma \mathrm{s}$, we then proceed as follows.

Instead of jointly fitting the astrophysical and cosmological parameters together for all the $15 \mathrm{C} i$ cosmologies, we fit only for the cosmological parameters $\gamma \mathrm{s}$ while keeping the astrophysical parameters fixed (except $\sigma$ ). We repeat the procedure 15 times, each time with the astrophysical parameters fixed to their best fitting values of each individual $\mathrm{C} i$ cosmology. This procedure is therefore equivalent to weighting every cosmology equally. For example, we fix the values of $\Pi_{c, 0}, \alpha$ and $\beta$ to their best-fitting values for $\mathrm{C} 1$ and then run MCMC analysis to find best-fitting values of $\gamma \mathrm{s}$. This analysis is repeated for all fifteen cosmologies, and therefore it gives us a set of fifteen best-fitting values of $\gamma_{h_{0}}, \gamma_{b}$ and $\gamma_{\sigma_{8}}$. The range of these cosmological parameters $\gamma_{\mathrm{s}}$ exceeds the pure statistical uncertainty of the fit, which is driven by the overall extremely large number of objects in our simulations and therefore does not reflect the underlying limitations of our modelling. The resulting range for both the astrophysical and cosmological MOR parameters are shown in Table 4 and 5 , respectively. The mean values quoted in these tables are simply the mean of the systematic uncertainty range. 
Table 2. The final number of haloes used in our analysis at different snapshots in different cosmological boxes above the lower mass limit $M_{\mathrm{vir}}>2 \times 10^{14} M_{\odot}$ (corresponding to approximately $10^{4}$ particles).

\begin{tabular}{ccccccccccccccccccccccc} 
z & C1 & C2 & C3 & C4 & C5 & C6 & C7 & C8 & C9 & C10 & C11 & C12 & C13 & C14 & C15 \\
\hline 0 & 487 & 1698 & 4639 & 2945 & 5063 & 2998 & 4864 & 7823 & 10454 & 12293 & 14103 & 17817 & 8711 & 21879 & 20912 \\
0.14 & 350 & 1335 & 3847 & 2299 & 4065 & 2262 & 3677 & 6150 & 8386 & 10093 & 11340 & 14570 & 6003 & 17818 & 16701 \\
0.29 & 219 & 910 & 2976 & 1641 & 3033 & 1465 & 2547 & 4601 & 6116 & 7729 & 8413 & 11172 & 3644 & 13550 & 12258 \\
0.47 & 121 & 516 & 2050 & 1034 & 2015 & 857 & 1460 & 3059 & 4102 & 5290 & 5607 & 7742 & 1861 & 9369 & 8087 \\
0.67 & 59 & 254 & 1271 & 553 & 1144 & 396 & 711 & 1694 & 2351 & 3297 & 3244 & 4744 & 754 & 5577 & 4576 \\
0.90 & 15 & 103 & 645 & 218 & 559 & 141 & 272 & 790 & 1091 & 1673 & 1558 & 2436 & 240 & 2872 & 2185 \\
\hline
\end{tabular}

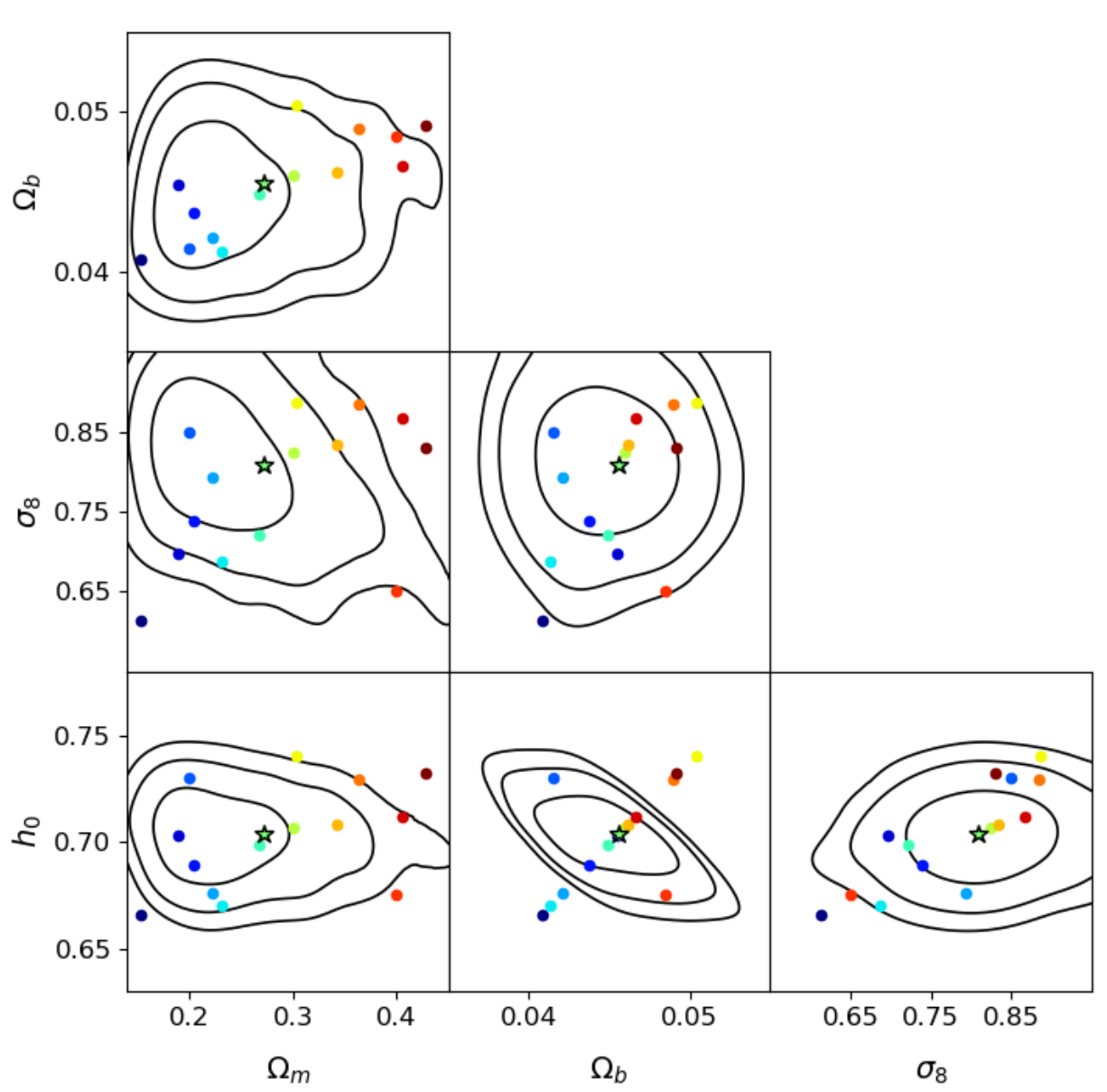

C1

Figure 1. Distribution of cosmological parameters for the fifteen cosmologies used in this paper. WMAP7 (i.e. our C8) is highlighted by star symbols. The black contours represent $68 \%, 95 \%$ and $99 \%$ confidence limits on these parameters (Bocquet et al. 2019) with additional Gaussian priors applied on $h_{0}$. 


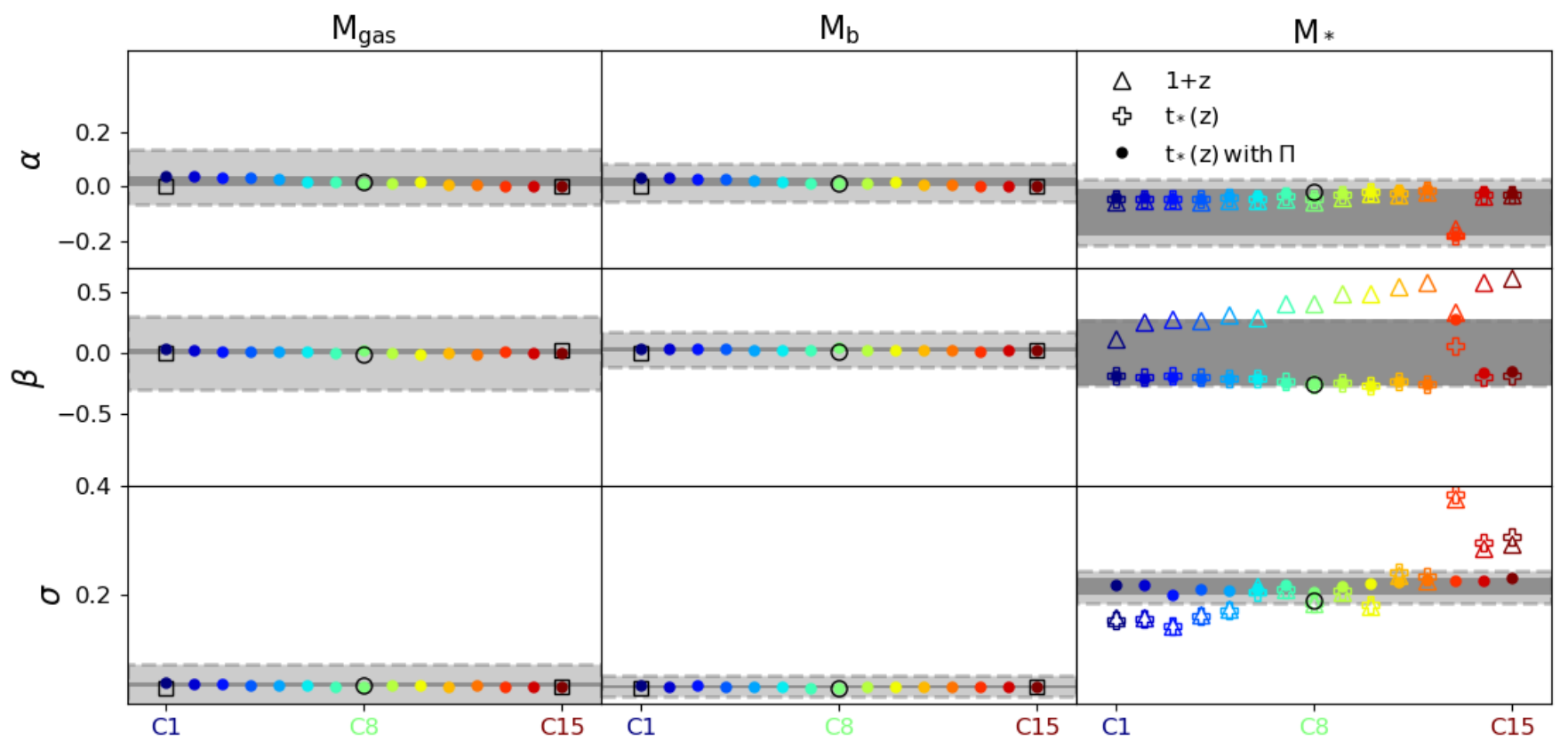

Figure 2. Variation in best-fitting values of $\alpha, \beta$ and $\sigma$ as a function of cosmology for $M_{\text {gas }}-M_{\mathrm{vir}}, M_{\mathrm{b}}-M_{\mathrm{vir}}$ and $M_{*}-M_{\mathrm{vir}}$ scaling relations. Filled circles correspond to the cosmologies shown in Figure 1 with the same color scheme. The lighter grey bands enclosed by dashed lines represent current state-of-the-art observational uncertainties (Bulbul et al. 2019, Chiu et al. 2018). Empty squares correspond to non-radiative runs (performed for $\mathrm{C} 1$ and $\mathrm{C} 15$ ) whereas empty circles correspond to the simulation run with a different feedback scheme for C8 cosmology. Darker grey bands represent the systematic uncertainty range quoted in Table 4

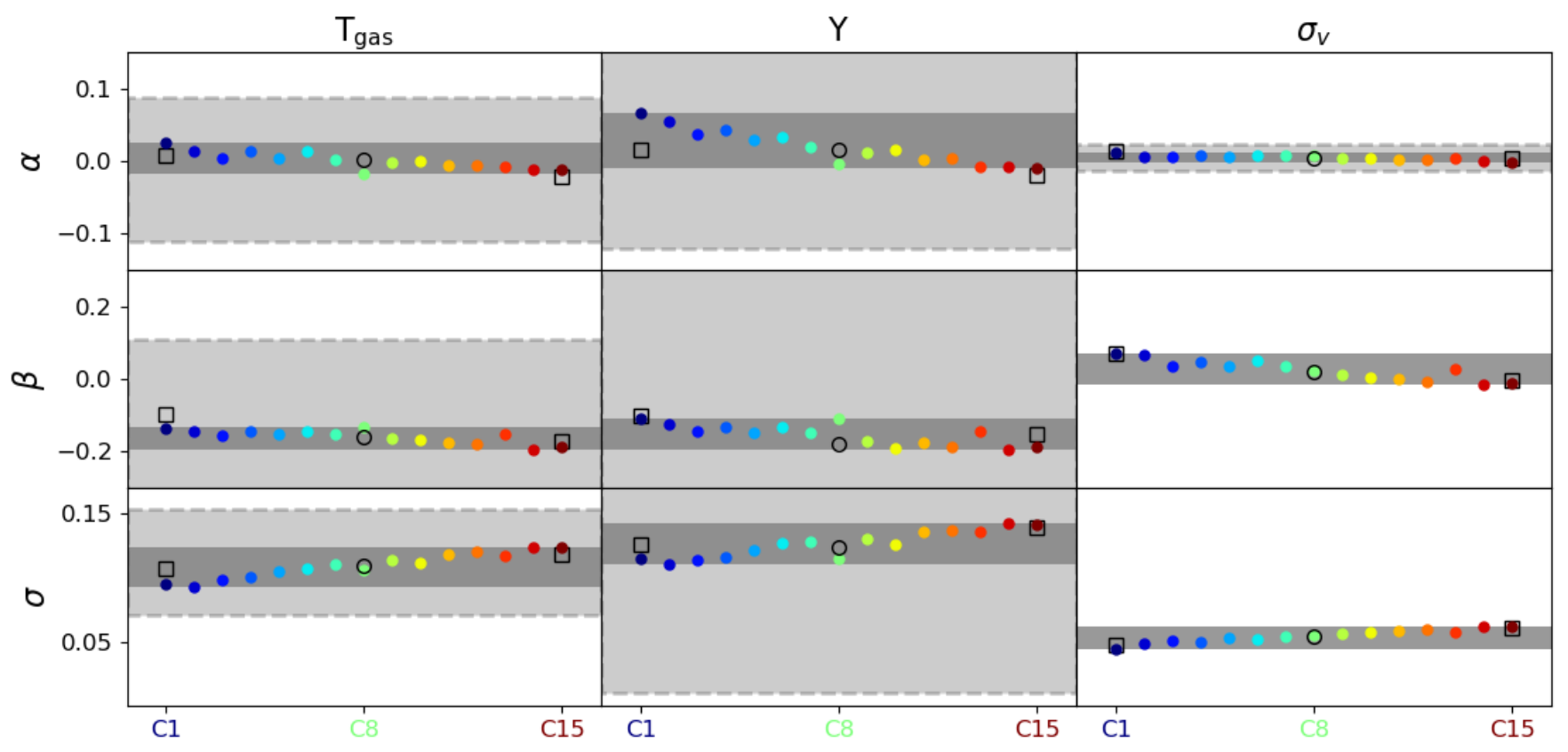

Figure 3. Same as Figure 2 for $T_{\text {gas }}-M_{\mathrm{vir}}, Y-M_{\mathrm{vir}}$ and $\sigma_{v}-M_{\mathrm{vir}}$ scaling relations. The uncertainty on $\sigma_{v}-M$ relation is the result of previous simulation studies (Evrard et al. 2008). 
Table 3. Self-similar mass $\left(\alpha_{s s}\right)$ and redshift $\left(\beta_{s s}\right)$ dependence of the MORs.

\begin{tabular}{ccccccc} 
& $M_{\text {gas }}$ & $M_{\mathrm{b}}$ & $M_{*}$ & $T_{\text {gas }}$ & $Y$ & $\sigma_{v}$ \\
\hline$\alpha_{s s}$ & 1 & 1 & 1 & $2 / 3$ & $5 / 3$ & $1 / 3$ \\
$\beta_{s s}$ & 0 & 0 & 0 & $2 / 3$ & $2 / 3$ & $1 / 3$ \\
\hline
\end{tabular}

\section{Results}

We present here our results for each of the four studied MORs. Note that, the observational results are generally at a different overdensity. Thus, the comparison of our results with the observations in this and the following sections is for qualitative purpose only.

\section{1 $M_{\text {gas }}-M_{\text {vir }}$ scaling relation}

For the $M_{\text {gas }}-M_{\text {vir }}$ scaling relation we obtain $\alpha$ and $\beta$ consistent with zero, i.e. no deviations from self-similarity. The log-normal scatter is $\lesssim 4 \%$. The derived self-similar redshift evolution and small intrinsic scatter are in agreement with observational constraints from B19. B19 find a steeper mass dependence $(\alpha \sim 0.26)$, while other observational results suggest a mass-slope consistent with self-similarity (e.g., Mantz et al. 2016). Note that, these observations are performed at $\Delta_{500 c}$. While here we extend to virial radius where the baryon fraction of massive systems, such as those considered here, is approximately the cosmological baryon fraction. Thus the gas fraction $\left(M_{\text {gas }} / M_{\text {vir }}\right)$ is approximately constant and independent of the cluster mass i.e. $\alpha=0$.

Among the cosmological parameters $\gamma \mathrm{s}$, we find that the $M_{\text {gas }}-M_{\text {vir }}$ scaling relation is consistent with being independent of $h_{0}$, and independent of $\sigma_{8}$ within $\sim 2 \sigma$. We find $M_{\text {gas }} \propto f_{b}^{0.8}$, which is slightly shallower but significantly away from the dependence expected from a closed box scenario (i.e. $M_{\text {gas }} \propto f_{b}$ ).

In the upper and lower panel of Figure 4 we show respectively the original $M_{\text {gas }}$ and the predicted $M_{\text {gas }}^{\prime}$ (the rescaled $M_{\text {gas }}$ at the pivot redshift and C8 pivot cosmology i.e. $M_{\text {gas }}$ - redshift dependence - cosmology dependence) as a function of $M_{\text {vir }}$ for all the clusters in our sample of all the 15 analyzed cosmologies. The solid and dashed grey lines in the lower panel correspond to the best-fit and $3 \sigma$ regions at the $\mathrm{C} 8$ cosmology. We note that the applied rescaling effectively removes the cosmological dependence of the $M_{\text {gas }}$ $-M_{\text {vir }}$ scaling relation.

\section{2 $\quad M_{\mathrm{b}}-M_{\mathrm{vir}}$ scaling relation}

For the $M_{\mathrm{b}}-M_{\mathrm{vir}}$ relation we find both $\alpha$ and $\beta$ close to zero and hence consistent with the self-similar scenario. The redshift evolution we find is therefore in agreement with current observational constraints (Chiu et al. 2018), while we find a mass-dependence slope shallower than Chiu et al. (2018). The log-normal scatter is also remarkably small $(\lesssim 3 \%)$. We stress however that the observational constraints from Chiu et al. (2018) have been derived within different clusterradii and for cluster samples in a different mass and redshift

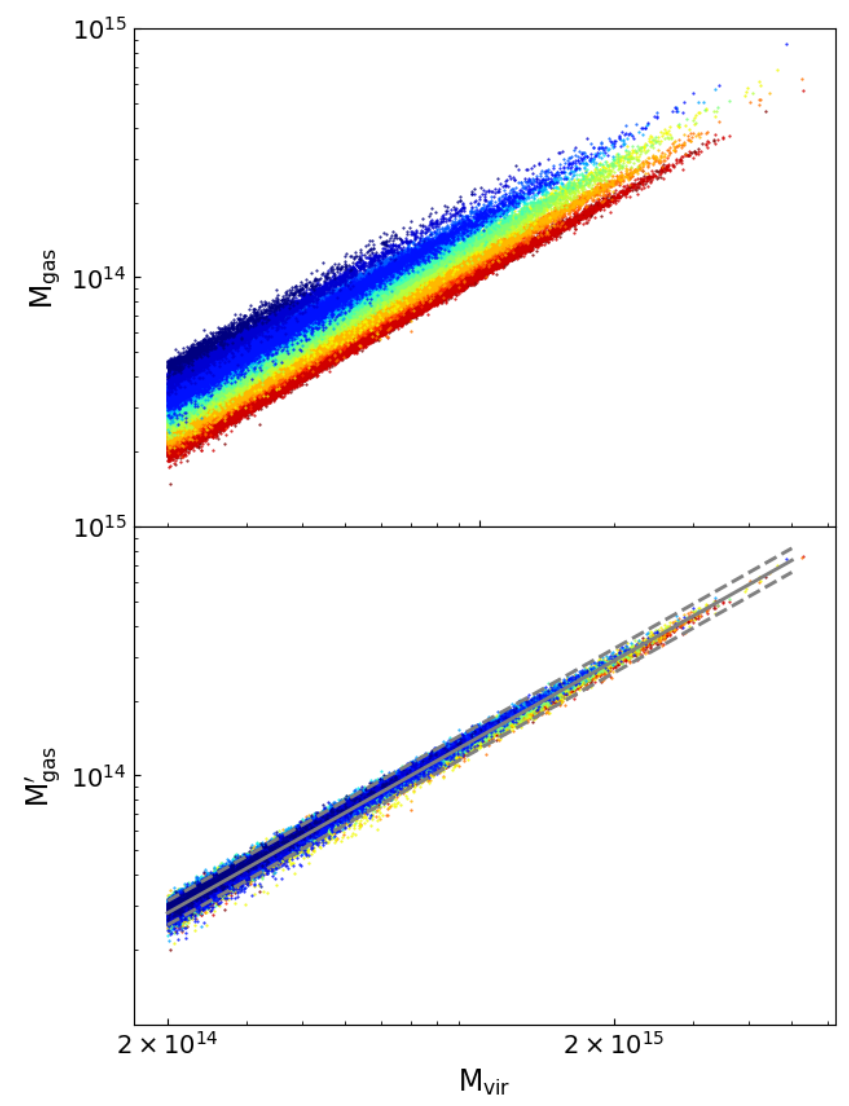

Figure 4. Top panel: Gas mass as a function of halo mass. The color scheme is same as in Figure 1 Bottom panel: Same as top panel after rescaling all data points to $\mathrm{C} 8$ cosmology and absorbing the predicted redshift evolution. The solid and dashed grey lines in the lower panel corresponds to the best-fit and $3 \sigma$ regions at the $\mathrm{C} 8$ cosmology.

range, and therefore a direct comparison is not straightforward.

For the cosmological MOR parameters $\gamma \mathrm{s}$, we find both $\gamma_{h_{0}}$ and $\gamma_{\sigma_{8}}$ consistent with zero, i.e. the $M_{\mathrm{b}}-M_{\text {vir }}$ relation does not significantly dependent on $h_{0}$ and $\sigma_{8}$. We find $M_{\mathrm{b}} \propto f_{b}^{0.87}$, close to, but still significantly different, than one (the expected closed-box scenario). More in detail, the total baryonic mass has a closer behaviour to the expected closedbox scenario than each individual $M_{\text {gas }}$ and $M_{*}$ component (section 4.3), consistent with the expected anti-correlation of $M_{\text {gas }}$ and $M_{*}$ at fixed halo mass (Wu et al. 2015). A more focused analysis on the correlation coefficients of different observables at fixed halo-mass will be presented in future works.

\section{3 $M_{*}-M_{\mathrm{vir}}$ scaling relation}

$M_{*}$ is the sum of mass of all star particles within a given overdensity radius. In the right-hand panel of Fig 2 we show the cosmology dependence of $\alpha, \beta$ and $\sigma$ for $M_{*}-M_{\text {vir }}$ scaling 
Table 4. Mean values and systematic uncertainties for astrophysical MOR parameters (described in detail in Section 3.1. The mean values quoted here are the mean of the systematic uncertainty range with symmetric error-bars.

\begin{tabular}{ccccccc} 
& $M_{\text {gas }}$ & $M_{\mathrm{b}}$ & $M_{*}$ & $T_{\text {gas }}$ & $Y$ & $\sigma_{v}$ \\
\hline$\Pi_{c, 0}$ & $31.4 \pm 0.4$ & $31.45 \pm 0.39$ & $28.07 \pm 1.41$ & $0.5 \pm 0.2$ & $31.9 \pm 0.2$ & $6.4 \pm 0.1$ \\
$\alpha$ & $0.02 \pm 0.02$ & $0.02 \pm 0.02$ & $-0.09 \pm 0.08$ & $0.0 \pm 0.02$ & $0.03 \pm 0.04$ & $0.01 \pm 0.07$ \\
$\beta$ & $0.01 \pm 0.02$ & $0.03 \pm 0.01$ & $0.003 \pm 0.275$ & $-0.16 \pm 0.03$ & $-0.15 \pm 0.04$ & $0.03 \pm 0.04$ \\
$\sigma$ & $<0.04$ & $<0.03$ & $0.22 \pm 0.02$ & $0.11 \pm 0.02$ & $0.13 \pm 0.02$ & $0.05 \pm 0.01$ \\
\hline
\end{tabular}

Table 5. Same as Table 4 for cosmological MOR parameters.

\begin{tabular}{ccccccc} 
& $M_{\text {gas }}$ & $M_{\mathrm{b}}$ & $M_{*}$ & $T_{\text {gas }}$ & $Y$ & $\sigma_{v}$ \\
\hline$\gamma_{h_{0}}$ & $-0.13 \pm 0.22$ & $0.02 \pm 0.21$ & $1.20 \pm 0.55$ & $0.78 \pm 0.05$ & $0.65 \pm 0.20$ & $0.38 \pm 0.04$ \\
$\gamma_{b}$ & $0.80 \pm 0.03$ & $0.87 \pm 0.03$ & $2.49 \pm 0.1$ & $-0.02 \pm 0.01$ & $0.78 \pm 0.03$ & $-0.05 \pm 0.01$ \\
$\gamma_{\sigma 8}$ & $-0.14 \pm 0.06$ & $-0.10 \pm 0.05$ & $1.71 \pm 0.28$ & $0.14 \pm 0.04$ & $0.01 \pm 0.05$ & $-0.01 \pm 0.01$ \\
\hline
\end{tabular}

relation (empty triangles). The dark grey shaded region is represents observational uncertainties taken from Chiu et al. (2018), centered at C8 cosmology (see their Table 3). Chiu et al. (2018) use DES and WISE/Spitzer data to constrain $M_{*}-M_{500 c}$ scaling relation for galaxy clusters in the redshift range $0.2<z<1.25$, with masses $M_{500 c} \gtrsim 2.5 \times 10^{14} M_{\odot}$. They assume a simple power law in $1+\mathrm{z}$ as a redshift dependence.

We find that $\alpha$ remains nearly independent of cosmology with $\alpha \sim 0$, close to the self similar prediction (except at $\mathrm{C} 13$ where it shows a small deviation from the selfsimilarity). However, both, the redshift dependence, $\beta$ and the log-normal scatter, $\sigma$ show a strong cosmology dependence. The most plausible explanation for the strong cosmology dependence seen in $\beta$ is that our simplistic assumption, $F(z)=1+z$ does not capture the redshift evolution of $M_{*}$ $-M_{\text {vir }}$ scaling relation.

The evolution of the stellar mass fraction of a galaxy is more directly linked to its stellar age rather than its redshift. Each star particle in the Magneticum simulations is described by a single stellar population (SSP) model, generated at a given redshift (expansion factor) and then passively evolved. From the post-processing analysis, we first define the age of the galaxy for each sub-halo, corresponding to the average expansion factor of all the associated star particles. We then average (weighted by the stellar mass of the galaxy) over the expansion factor of all galaxies residing in each main halo to obtain an average redshift of the formation of the stellar content of the cluster. At a given snapshot, the difference between the age of the Universe at that snapshot and the age of the Universe at the formation time of the stellar content represents the stellar age of the halo, $t_{*}(z)$.

Using $t_{*}(z)$ (shown by empty plus markers) instead of $1+z$ (empty triangles) removes most of the cosmology dependence present in $\beta$ as shown in Fig 2, thus supporting the idea that the time evolution of the galaxy population is a better description. However, this parametrization does not help with the cosmology dependence of the scatter $\sigma$. In Fig 5, we show $\sigma$ as a function of $\Pi_{c}$, i.e. the log normalization of the scaling relation. The black-solid line in the figure follows,

$$
\sigma=\sigma_{0} \exp \left[-0.42\left(\Pi_{c}-\Pi_{c, 0}\right)\right]
$$

where, $\sigma_{0}=0.21$ and $\Pi_{c, 0}=28.69$. This figure suggests that the normalization and the scatter in $M_{*}-M_{\text {vir }}$ scaling relation are tightly correlated, and both of them have similar cosmology dependence. Therefore, instead of further expanding the scatter $\sigma$ as a complicated function of cosmological parameters, we choose to write it a simple function of $\Pi_{c}$. As shown in Fig 2 (filled circles), the combination of $t_{*}(z)$ redshift evolution and $\sigma\left(\Pi_{c}\right)$ gives us the desired form of $M_{*}-M_{\text {vir }}$ scaling relation.

Consistently with Chiu et al. (2018), we find that the mass slope is smaller than one (i.e. $\alpha+\alpha_{s s} \sim 0.9$ ). A direct comparison with the redshift evolution is limited by the different adopted functional form and observed overdensities, but it is in general consistent with a mild evolution, at least within the probed redshift range. Within the adopted parametrization of the scatter, we find a residual log-normal scatter $\sigma$ close to $20 \%$. With respect to the cosmological parameters, we find $\gamma_{h_{0}}$ consistent with zero given the large systematic uncertainty. On the other hand, we find $M_{*} \propto f_{b}^{2.56}$ and $\sigma_{8}^{1.77}$ (with large uncertainty on $\gamma_{\sigma_{8}}$ ). This is not too surprising, since star formation is regulated by much more complex physical processes than, for example, the physics describing the relationship between mass and velocity dispersion. Stellar mass is the outcome of physics acting at small scales, such star formation and feedback. At the same time, it is directly affected by large scale cosmology dependent processes, such as cosmological infall and mergers. Therefore, the cosmology dependence of $M_{*}$ can be strong and non-trivial as suggested by our results. 


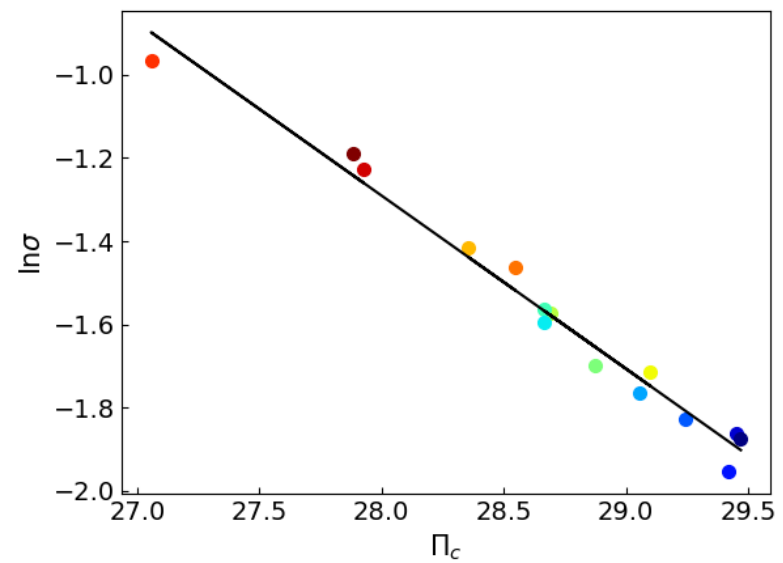

Figure 5. Correlation between scatter and log-normalization of stellar mass scaling relation. Solid-black line follows Equation 4

\section{4 $T_{\text {gas }}-M_{\text {vir }}$ scaling relation}

For the $T_{\text {gas }}-M_{\text {vir }}$ relation, we find a good agreement with the self-similar expectation in the mass slope (e.g., $\alpha \sim$ 0 ), consistent with many observational studies (Vikhlinin et al. 2009 Arnaud, Pointecouteau \& Pratt 2005 Mantz et al. 2016). However note that, B19 find a steeper mass slope compared to the self-similar prediction. We find a small negative redshift evolution $(\beta \sim-0.16)$ consistent within 1- $\sigma$ with observed redshift evolution (eg. Mantz et al. 2016, B19). We find a log-normal scatter of $11 \%$, consistent (within $2-\sigma$ ) with the observed scatter in $T_{\text {gas }}-M_{500 c}$ relation (Bulbul et al. 2019).

For the cosmology dependence of the scaling relation, we find $T_{\text {gas }} \propto h_{0}^{0.78} f_{b}^{-0.02} \sigma_{8}^{0.14}$. Therefore, the temperaturemass scaling relation is almost independent of the baryon fraction and $\sigma_{8} . T_{\text {gas }}$ has a significant dependence on $h_{0}$, consistent with the theoretical expectation of $2 / 3$ if $T_{\text {gas }}$ $\propto \rho_{c}^{1 / 3}$ (and $\rho_{c} \propto h_{0}^{2}$ ). The $h_{0}$ dependencies of $T_{\text {gas }}$ and $\sigma_{v}$ can also be described by their dependencies on the halo mass, whereas, it is not the case for other observables (see Table 5). However, the $h_{0}$ dependencies of most of the observables are well described by their dependencies on $\rho_{c}$. We further highlight that the cosmology dependence of MOR normalization i.e. $\gamma$-parameters represent more an "effective cosmology dependence" and could incorporate the cosmological dependence of other quantities.

\section{5 $Y-M_{\mathrm{vir}}$ scaling relation}

The X-ray integrated pseudo-pressure $Y$ is an observable which is characterized by a relatively low intrinsic scatter and its closely connected to the SZE observable (Kravtsov, Vikhlinin \& Nagai 2006 Nagai 2006, Nagai, Kravtsov \& Vikhlinin 2007; Bonamente et al. 2008 | Vikhlinin et al. 2009. Anderson \& Bregman 2011, Benson et al. 2013; Mantz et al. 2015 B19). The self-similar scenario predicts $Y \propto M_{\mathrm{vir}}^{\mathrm{Jj}}$ $F(z)^{2 / 3}$. We find $\alpha \sim 0$, consistent with the observations by Vikhlinin et al. (2009); Lovisari, Reiprich \& Schellenberger (2015); Mantz et al. (2016), whereas it is shallower than the observations by Arnaud, Pointecouteau \& Pratt (2007) and
B19. We find $\beta \sim-0.15$, i.e. a small deviation from selfsimilarity in the redshift dependence as expected from the results for $T_{\text {gas }}-M_{\text {vir }}$ relation. Observed redshift evolution of $Y$-mass relation is consistent with zero given the large uncertainties (eg. Mantz et al. 2016, B19). The scatter in $Y$ $-M_{\text {vir }}$ relation is around $13 \%$, driven by the scatter in $T_{\text {gas }}$ $-M_{\text {vir }}$ relation, and consistent with the observed scatter in $Y-M_{500 c}$ relation (Bulbul et al. 2019).

In case of cosmology dependence we find, $Y \propto f_{b}^{0.78}$, driven by the strong baryon fraction dependence of gas mass, $Y \propto h_{0}^{0.65}$, driven by its temperature dependence and no dependence on $\sigma_{8}$.

\section{6 $\sigma_{v}-M_{\mathrm{vir}}$ scaling relation}

For perfectly virialized objects, velocity dispersion is tracing the total halo mass, since it is shaped by gravity only. Previous simulations studies (eg. Evrard et al. 2008, Saro et al. 2013 Munari et al. 2013) have already shown that threedimensional $\sigma_{v}-M_{\text {vir }}$ relation stays close to the self-similar prediction. We also find $\alpha$ and $\beta \sim 0$. Consistent with previous results, we find a remarkably small scatter in the scaling relation $(\sim 5 \%)$. Previous studies have shown, however, that the one-dimensional velocity dispersion-mass relation has a significantly larger scatter, due to halo triaxiality (White, Cohn \& Smit 2010, Saro et al. 2013).

Coming to the cosmology dependence, we find $\sigma_{v} \propto$ $h_{0}^{0.38}$ and to be independent of baryon fraction and $\sigma_{8}$, again in agreement with a self-similar scenario (since $\sigma_{v}^{2} \propto \rho_{c}^{1 / 3}$ ).

\section{Implication for cosmological studies}

In this section, we forecast the impact of the results presented in the previous section for the parameterization of the MOR on an idealized cluster number-counts cosmology experiment. The simulated experiment resembles a simplified eROSITA cluster cosmology analysis, with an idealized gasmass selected cluster catalog over $15,000 \mathrm{deg}^{2}$. This analysis does not capture all the sophisticated modelling of the eROSITA selection function (e.g., Grandis et al. 2018), but has solely the purpose of highlighting the impact of different cosmological parametrization of the MOR for clustercosmology experiments.

The mock catalog is generated using the Tinker et al. (2008) halo-mass function assuming a WMAP7 cosmology (Komatsu et al. 2011). Gas-masses are then computed from the total cluster mass (including intrinsic scatter) using equations 1 and 2 and Tables 4 and 5 The final catalog consists in all clusters with final $\log \left(M_{\text {gas }} / M_{\odot}\right)>13.75$ (corresponding to a halo mass $\sim 2 \times 10^{14} M_{\odot}$ ) between redshift range $0.1-1$.

We then analyze the above sample, by computing the likelihood of observing the number of clusters $N_{i, j}$ in the survey area for a given redshift bin $i$ and $M_{\text {gas }}$ bin $j \mathcal{L}\left(N_{i, j} \mid \vec{\theta}\right)$, where $\vec{\theta}$ contains both the MOR and the cosmological parameters. In details, we assume a Gaussian likelihood of the form:

$$
\mathcal{L}(d \mid \theta) \propto \frac{\exp \left[-\frac{1}{2}(d-m(\vec{\theta}))^{T} C^{-1}(d-m(\vec{\theta}))\right]}{\sqrt{(2 \pi)^{M} \operatorname{det}(C)}} .
$$


Table 6. Priors used in our analysis while forecasting the impact of our MOR parameterization on an idealized eROSITA-like experiment (see Section 5 for details). $\mathcal{U}(a, b)$ represents uninformative prior in the range $(a, b) . \mathcal{N}(\mu, \sigma)$ represents Gaussian prior with mean $\mu$ and width $\sigma$. The priors on $\Omega_{m}, h_{0}, \Omega_{b} h_{0}^{2}$ and $\ln A_{s}$ are same for all three cases (therefore not shown in table) are given in Section 5
$\Pi_{c}$
$\alpha$
$\beta$
$\sigma$
$\gamma_{h_{0}}$
$\gamma_{b}$
$\gamma_{\sigma_{8}}$

\begin{tabular}{|c|c|c|c|c|c|c|c|}
\hline case-(i) & $\mathcal{N}(31.40,0.157)$ & $\mathcal{N}(0.02,0.13)$ & $\mathcal{N}(0.01,0.192)$ & $\mathcal{N}(0.10,0.05)$ & - & - & - \\
\hline case-(ii) & $"$ & $"$ & $"$ & $"$ & $\mathcal{N}(-0.13,0.22)$ & $\mathcal{N}(0.80,0.03)$ & $\mathcal{N}(-0.14,0.06)$ \\
\hline case-(iii) & $\mathcal{N}\left(31.40-\ln \left(10^{\Delta\left(\Omega_{m}\right)}, 0.157\right)\right.$ & $"$ & $"$ & $"$ & $"$ & $"$ & $"$ \\
\hline
\end{tabular}

where $M$ is the dimensionality of the data vector (three redshift bins $\times$ five gas mass bins $=15), C$ is the covariance matrix, and $d$ and $m(\vec{\theta})$ are respectively the number counts data vector and the corresponding expectation values for the set of parameters $\vec{\theta}$. The covariance matrix is defined as the sum of a Poisson and sample variance contribution and it is computed analytically at each step of the chain. The two components of the covariance matrix are,

$$
C^{\text {Poisson }}=\delta_{\mathrm{ii}}\langle N\rangle_{i}
$$

and

$$
C_{\mathrm{ij}}^{\mathrm{Samp} \text { Var }}=\langle b N\rangle_{i}\langle b N\rangle_{j} \sigma^{2}\left(V_{i}, V_{j}\right) .
$$

where $\langle N\rangle_{i}$ is the expected number of cluster in the $i$-th bin, while $b$ is the linear halo bias (Tinker et al. 2010). The last term of equation corresponds to the rms variance of the linear density field within the comoving volume $V_{i}$, which we approximate with a top-hat window symmetric around the azimuthal axis. We refer the reader to Costanzi et al. (2019) for further details on the likelihood and covariance matrix calculation.

The parameter space $\vec{\theta}$ is explored with the affine invariant sampler emcee (Foreman-Mackey et al. 2013).

More in detail, the parameters varied during the MCMC analysis are $\Pi_{c, 0}, \alpha, \beta, \sigma$ and the cosmological parameters (within a flat $\Lambda$ CDM model) $\Omega_{m}, h_{0}, \Omega_{b} h^{2}$ and $\ln A_{s}$. We apply Gaussian priors on $h_{0}(0.7 \pm 0.05), \Omega_{b} h^{2}(0.02208 \pm$ $0.00052)$, and uninformative priors on $\Omega_{m}(0.05,0.5)$, and $\ln A_{s}(1.0,6.0)$. We also apply Gaussian priors on $\Pi_{c}(31.40 \pm$ $0.157), \alpha(0.02 \pm 0.130)$ and $\beta(0.01 \pm 0.192)$, where the mean values of the Gaussian are taken from our Table 4 , and uncertainties are from the estimated uncertainties on the $L_{x}-M$ from Grandis et al. (2018) (rescaled appropriately in case of $\alpha$ ). We apply a Gaussian normal prior on the intrinsic scatter $\sigma_{\ln } M_{\text {gas }}=0.10 \pm 0.05$ (the scatter parameter includes the intrinsic and observational scatter) based on the constraint on the scatter of the gas mass-halo mass relation from Mantz et al. (2016).

We explore three different scenarios for a cosmological analysis. In all this three cases, we use the above mentioned priors, summarized in Table 6

(i) Fixing $\gamma$ 's to zero: This analysis describes the case where the normalization of the MOR is assumed to be cosmology independent, which represents the approach typically adopted in the literature. In this case, the amplitude of MOR is simply $\Pi_{c}=\Pi_{c, 0}$. We recover the input param- eters in an unbiased way as shown by the grey contours in Fig 6 .

(ii) Varying $\gamma$ 's: In this case, we also include the cosmological dependence on the MOR, as discussed in Section 3. We now also include $\gamma$ 's as free parameters, with associated Gaussian prior with mean and width taken from Table 5 As a result, the marginalized posterior distributions exhibit now a stronger degeneracy between $\Pi_{c, 0}$ and cosmological parameters as shown by red contours in Fig 6 For example, now $\Pi_{c, 0}$ shows a strong anti-correlation with $f_{b}$, driven by the positive value of $\gamma_{b}$ without any significant variation in the one-dimensional posteriors (as expected due to the uninformative prior on $\left.\Pi_{c, 0}\right)$. Such degeneracies otherwise cannot be tested with observational data. Note that the correlation between $\Pi_{c, 0}$ and $h_{0}$ is induced by the tight Gaussian prior applied on $\Omega_{b} h_{0}^{2}$ and the anti-correlation between $\Pi_{c, 0}$ and $f_{b}$.

(iii) Varying $\gamma$ 's + weak lensing cosmological dependencies on $\Pi_{c}$ : In the previous case, we obtained the same marginalized posterior distributions on the cosmological parameters, as expected. The combination of multiple cosmological observables has the potential of breaking degeneracies and provide tighter constraints. Therefore, we now explore the possibility of combining different observables with different sensitivity to the cosmological parameters through their MOR.

In a real scenario, the amplitude of the gas-mass relation is calibrated with weak-lensing mass estimates. However, weak-lensing calibration also has a cosmological dependence. Simet et al. (2017) derived matter density dependent mass-richness scaling relation for redMaPPer cluster catalog using weak-lensing data from SDSS. Within their adopted model, the amplitude of the scaling relation depends only on the assumed value of $\Omega_{M}$ (in a range $\sim 0.26$-0.34) with a linear decline of log-amplitude as $\Omega_{M}$ increases. We use their weak-lensing mass calibration to model the prior of our MOR. The amplitude of $M_{\text {gas }}-M_{\text {halo }}$ relation can now be written as,

$$
\Pi_{c}\left(\Omega_{m}\right)=\Pi_{c}-\ln 10^{\Delta\left(\Omega_{m}\right)}
$$

where, $\Delta\left(\Omega_{m}\right)=\alpha \frac{d \log M_{\mathrm{WL}}}{d \Omega_{m}}\left(\Omega_{M}-0.3\right)$. Incorporating above dependencies modifies the mean of the Gaussian prior applied on $\Pi_{c}$ to $31.40-\ln 10^{\Delta\left(\Omega_{m}\right)}$, whereas, the width of the Gaussian remains unchanged. We measure the gas-mass and the weak lensing mass, assuming a reference cosmology and then calibrate the normalization of the gas-mass relation at that reference cosmology. We then explore the cosmological parameter space at each new cosmology. We rescale the 
gas-mass relation (assuming our MOR), and the constraints we obtain from weak lensing as if we were at the new cosmology. The results of the analysis are shown by blue contours in Fig 6 Addition of priors from weak-lensing experiments does not impact most of the model parameters due to the different cosmological dependencies of the weak-lensing (which is mostly sensitive to $\Omega_{m}$ ) and $M_{\text {gas }}-M_{\text {vir }}$ scaling relation (which is proportional to the baryon fraction). However, there is a slight improvement in the constraints on $\Omega_{m}$ (from $0.279_{-0.051}^{+0.059}$ in the previous case to $0.278_{-0.046}^{+0.046}$ ).

To compare the performance of the above described models, we use Deviance Information Criterion (DIC) and interpret the results using Jeffrey's scale. For a given model $M_{i}$, DIC is defined as (Spiegelhalter et al. 2002),

$$
\operatorname{DIC}\left(M_{i}\right)=\left\langle\chi^{2}\right\rangle+p_{d},
$$

where $\left\langle\chi^{2}\right\rangle=-2\left\langle\ln \mathcal{L}\left(d \mid \theta, M_{i}\right)\right\rangle$ is the mean of $\chi^{2}(\theta)$ and the term $p_{d}$ is called Bayesian complexity defined as, $p_{d}=$ $\left\langle\chi^{2}\right\rangle-\chi^{2}(\tilde{\theta})$, where $\tilde{\theta}$ is the maximum likelihood (minimum $\left.\chi^{2}\right)$ point. A model which describes the data vector better has a lower value $p_{d}$ and a higher likelihood and thus a lower value of DIC (Grandis et al. 2016).

For the three scenarios considered in this paper we find, $\operatorname{DIC}\left(M_{2}\right)-\operatorname{DIC}\left(M_{1}\right)=-4.1, \operatorname{DIC}\left(M_{3}\right)-\operatorname{DIC}\left(M_{1}\right)=-4.5$ and $\operatorname{DIC}\left(M_{3}\right)-\operatorname{DIC}\left(M_{2}\right)=-0.4$. Therefore, both the models with non-zero values of $\gamma \mathrm{s}$ (Model 2 and Model 3) have a positive preference over Model 1 . However, our final model Model 3 including priors from WL studies has an insignificant preference over model Model 2.

To summarize, our Model 1 represents a typical approach where the amplitude of the scaling relation is assumed to be independent of the cosmological parameters. Model 2 is based on our suggested MOR parameterization, which we find a more appropriate choice as it now includes correlations between the MOR normalization and cosmological parameters and therefore better represents our derived theoretical priors. We stress that, by design, the parametrization adopted in Model 2 does not weaken the constraining power of the data on the marginalized posteriors. Finally, Model 3 represents an idealized case to demonstrate how the combination of different observables (which are subject to different degeneracies) can exploit the theoretical priors we derived. This scenario has thus the potentiality of breaking these degeneracies in order to provide tighter cosmological constraints.

\section{Summary}

Magneticum simulations provide one of the most powerful tools for exploring large scales in the presence of both gravitational and complex hydro-dynamical processes in different cosmological environments. In this paper, we studied the mass-observable scaling relation for clusters $\left(M_{\mathrm{vir}}>\right.$ $\left.2 \times 10^{14} M_{\odot}\right)$ up to redshift $z<1$. We examined the cosmology dependence of the $M_{\text {gas }}-M_{\text {vir }}, T_{\text {gas }}-M_{\text {vir }}, \sigma_{v}-M_{\text {vir }}$ and $Y-M_{\text {vir }}$ relations using fifteen large cosmological boxes produced with the Magneticum simulation set-up with varying cosmological parameters. These cosmologies are chosen using Latin hypercube sampling to fairly sample the constraints on $h_{0}, \sigma_{8}$ and $\Omega_{m}$, obtained from the latest SPT cluster number count results. We divide the MOR parameters into two categories: $i$ ) the astrophysical ones, describing the normalization $\left(\Pi_{c, 0}\right)$, the mass-slope $(\alpha)$, the redshift evolution $(\beta)$, and the intrinsic lognormal scatter $(\sigma)$, and ii) the cosmological ones $\left(\gamma_{h_{0}}, \gamma_{b}\right.$ and $\left.\gamma_{\sigma_{8}}\right)$, describing respectively the impact of $h_{0}$, the baryon fraction $f_{b}$ and $\sigma_{8}$, on the amplitude of the scaling relations.

All four observables considered here show a perfect selfsimilar mass dependence i.e. $\alpha$ consistent with zero. The redshift dependence of $M_{\text {gas }}$ and $\sigma_{v}$ are in good agreement with the self-similar prediction whereas $T_{\text {gas }}$ and $Y$ show a small deviation. The scatter in $M_{\text {gas }}-M_{\text {vir }}$ relation is smallest (3-4\%) among the four observables followed by $\sigma_{v}(\sim 5 \%)$, $T_{\text {gas }}(\sim 11 \%)$ and $Y(\sim 13 \%)$. We investigate the cosmology dependence of $\alpha, \beta$ and $\sigma$, and do not find any significant variation, a result that therefore justifies our assumed functional form for the adopted scaling relations.

With respect to the cosmological parameters, we find that the $h_{0}$ dependence of the MOR agrees with the theoretical expectation, where the scaling of the observables is associated with the variation of the critical density of the Universe (as a function of both redshift and cosmology).

We find $M_{\text {gas }} \propto f_{b}^{0.8}$, i.e. the gas mass - halo mass scaling relation is slightly shallower but significantly away from the value expected from a closed box scenario $\left(M_{\text {gas }} \propto\right.$ $f_{b}$ ) given the uncertainty in $\gamma_{b} . T_{\text {gas }}$ and $\sigma_{v}$ are instead found to be independent of $f_{b}$, whereas, $Y \propto f_{b}^{0.78}$ driven by the baryon fraction dependence of the gas mass.

The cosmological dependence on $\sigma_{8}$ is consistent with zero for all the four studied observables.

In order to provide robust uncertainties on the MOR parameters (more reliable than the negligible statistical ones), we estimate the systematic uncertainty of the MOR by propagating the error associated with our choice of the functional form used to describe the scaling relations.

As a proof of concept, we show the impact of the cosmological dependence of the MOR for an idealized eROSITAlike cluster cosmology experiment. More in detail, we show that our cosmology dependent parametrization introduces a strong degeneracy between the amplitude of the scaling relation and the cosmological parameters, without affecting the one-dimensional marginalized posterior distribution and it is preferred over the cosmology independent normalization model. However, the combination of different observables, which are subject to different cosmological dependencies, can help in breaking these degeneracies and therefore provide a powerful way to tighten cosmological constraints.

Upcoming next generation surveys will allow us to calibrate the astrophysical parameters of MORs with unprecedented accuracy. While multi-wavelength data-sets are fundamental to directly constrain the astrophysical parameters from observations, the calibration of the cosmology dependence of the MOR is only possible through the analysis of cosmological hydro-dynamical simulations or accurate theoretical modelling of cluster formation and evolution. This work represents therefore a step towards understanding the impact of the cosmological dependence of galaxy cluster scaling relations with hydro-dynamical simulations for future cluster cosmology experiments.

\section{ACKNOWLEDGEMENTS}

We thank the anonymous referee for many insightful 


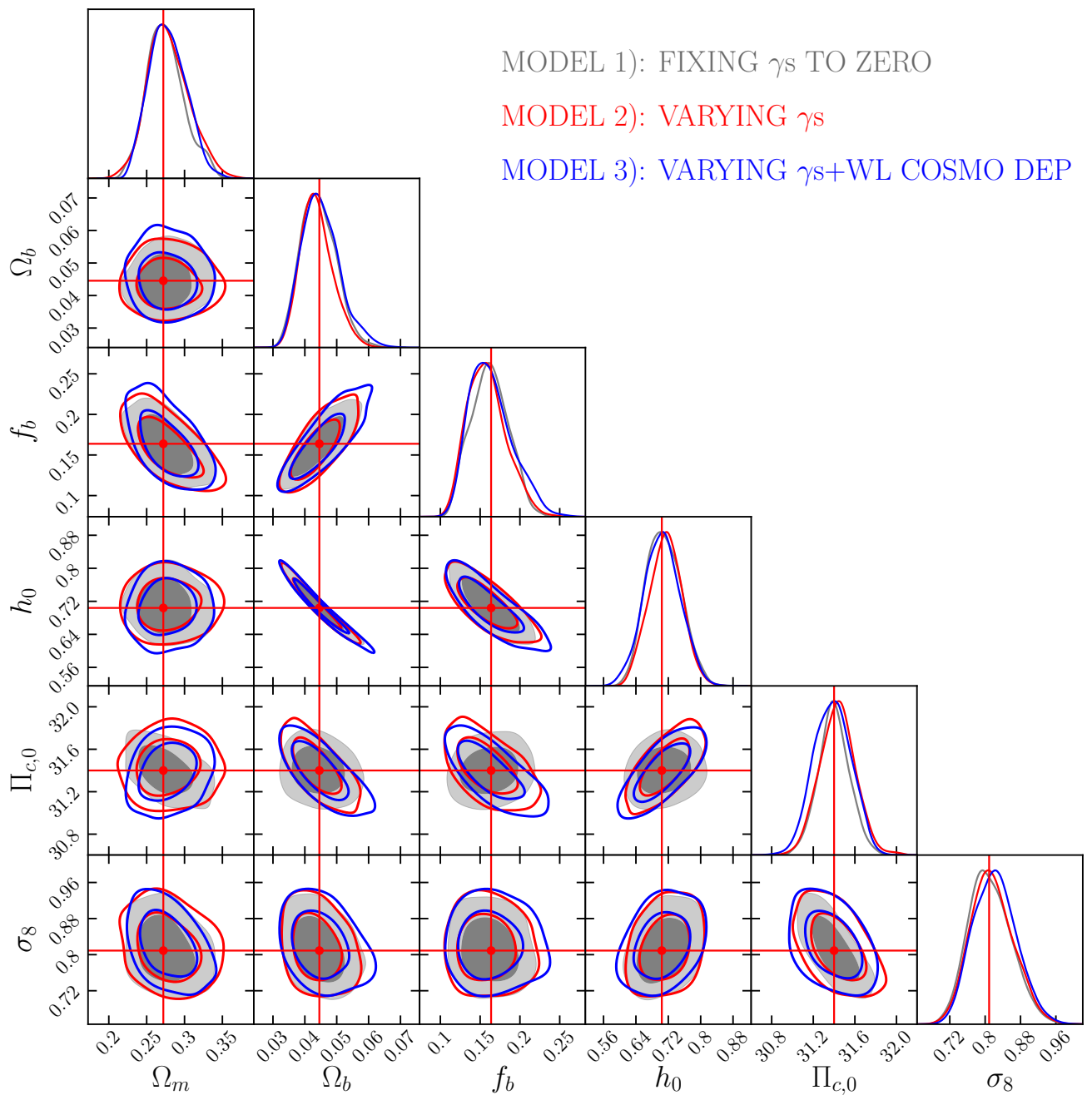

Figure 6. The $68 \%$ and $95 \%$ confidence limit contours for the scaling relation and cosmological parameters. In grey are the constraints for $\gamma_{\mathrm{s}}$ fixed to zero, in red the constraints including $\gamma_{\mathrm{s}}$ in the analysis, and in blue the constraints including $\gamma_{\mathrm{s}}$ and weak-lensing mass calibration to model the prior on the amplitude of the MOR.

suggestions and comments. We thank Sebastian Bocquet, Stefano Borgani, August Evrard, Salman Habib, Joseph Mohr, Daisuke Nagai and Elena Rasia for stimulating discussions and their valuable suggestions. PS, MC, AS are supported by the ERC-StG ClustersXCosmo grant agreement 716762 , AS is supported by the FARE-MIUR grant 'ClustersXEuclid' R165SBKTMA. Computations have been performed at the Leibniz-Rechenzentrum with CPU time assigned to the Project pr83li and "pr74d0". KD acknowledges support through ORIGINS, founded by the Deutsche Forschungsgemeinschaft (DFG, German Research Foundation) under Germany's Excellence Strategy EXC-2094 390783311 and by the DAAD, contract number 57396842 .

\section{REFERENCES}

Allen S. W., Evrard A. E., Mantz A. B., 2011, ARA\&A, 49, 409 Anderson M. E., Bregman J. N., 2011, ApJ, 737, 22

Applegate D. E. et al., 2014, MNRAS, 439, 48
Arnaud M., Pointecouteau E., Pratt G. W., 2005, A\&A, 441, 893

Arnaud M., Pointecouteau E., Pratt G. W., 2007, A\&A, 474, L37

Avestruz C., Lau E. T., Nagai D., Vikhlinin A., 2014, ApJ, 791, 117

Bardeau S., Soucail G., Kneib J. P., Czoske O., Ebeling H., Hudelot P., Smail I., Smith G. P., 2007, A\&A, 470, 449

Becker M. R., Kravtsov A. V., 2011, ApJ, 740, 25

Benson B. A. et al., 2013, ApJ, 763, 147

Biffi V. et al., 2016, ApJ, 827, 112

Biffi V., Dolag K., Merloni A., 2018, MNRAS, 481, 2213

Bocquet S. et al., 2019, ApJ, 878, 55

Bocquet S., Saro A., Dolag K., Mohr J. J., 2016, MNRAS, 456, 2361

Bocquet S. et al., 2015, ApJ, 799, 214

Böhringer H., Dolag K., Chon G., 2012, A\&A, 539, A120

Bonamente M., Joy M., LaRoque S. J., Carlstrom J. E., Nagai D., Marrone D. P., 2008, ApJ, 675, 106

Bryan G., Norman M., 1998, ApJ, 495, 80

Bulbul E. et al., 2019, ApJ, 871, 50

Capasso R. et al., 2019a, MNRAS, 486, 1594

Capasso R. et al., 2019b, arXiv e-prints, arXiv:1910.04773

Capasso R. et al., 2019c, MNRAS, 482, 1043 
Chiu I. et al., 2018, MNRAS, 478, 3072

Costanzi M. et al., 2019, MNRAS, 488, 4779

Cui W., Borgani S., Murante G., 2014, MNRAS, 441, 1769

DeRose J. et al., 2019, ApJ, 875, 69

Despali G., Giocoli C., Angulo R. E., Tormen G., Sheth R. K.,

Baso G., Moscardini L., 2016, MNRAS, 456, 2486

Di Matteo T., Colberg J., Springel V., Hernquist L., Sijacki D., 2008, ApJ, 676, 33

Dietrich J. P. et al., 2019, MNRAS, 483, 2871

Dolag K., Borgani S., Murante G., Springel V., 2009, MNRAS, 399, 497

Dolag K., Mevius E., Remus R.-S., 2017, Galaxies, 5, 35

Evrard A. E. et al., 2008, ApJ, 672, 122

Fabian A. C., 2010, in IAU Symposium, Vol. 267, Co-

Evolution of Central Black Holes and Galaxies, Peterson B. M.,

Somerville R. S., Storchi-Bergmann T., eds., pp. 341-349

Foreman-Mackey D., Hogg D. W., Lang D., Goodman J., 2013, PASP, 125, 306

Grandis S., Mohr J. J., Dietrich J. P., Bocquet S., Saro A., Klein

M., Paulus M., Capasso R., 2018, arXiv e-prints

Grandis S., Rapetti D., Saro A., Mohr J. J., Dietrich J. P., 2016,

MNRAS, 463, 1416

Gupta N. et al., 2016, ArXiv e-prints

Haardt F., Madau P., 2001, in Clusters of Galaxies and the High Redshift Universe Observed in X-rays, Neumann D. M., Tran

J. T. V., eds., p. 64

Hasselfield M. et al., 2013, JCAP, 7, 8

Heitmann K. et al., 2016, ApJ, 820, 108

Hilton M. et al., 2018, ApJS, 235, 20

Hirschmann M., Dolag K., Saro A., Bachmann L., Borgani S., Burkert A., 2014, MNRAS, 442, 2304

Hoekstra H., Herbonnet R., Muzzin A., Babul A., Mahdavi A.,

Viola M., Cacciato M., 2015, MNRAS, 449, 685

Hoekstra H., Mahdavi A., Babul A., Bildfell C., 2012, MNRAS, 427, 1298

Khedekar S., Churazov E., Kravtsov A., Zhuravleva I., Lau

E. T., Nagai D., Sunyaev R., 2013, MNRAS, 431, 954

Komatsu E. et al., 2011, ApJS, 192, 18

Kravtsov A. V., Vikhlinin A., Nagai D., 2006, ApJ, 650, 128

Lau E. T., Kravtsov A. V., Nagai D., 2009, ApJ, 705, 1129

Lau E. T., Nagai D., Kravtsov A. V., 2010, ApJ, 708, 1419

Lau E. T., Nagai D., Nelson K., 2013, ApJ, 777, 151

Lawrence E. et al., 2017, ApJ, 847, 50

Lovisari L., Reiprich T. H., Schellenberger G., 2015, A\&A, 573, A118

Mantz A. B., Allen S. W., Morris R. G., Rapetti D. A., Applegate D. E., Kelly P. L., von der Linden A., Schmidt R. W., 2014, MNRAS, 440, 2077

Mantz A. B. et al., 2016, MNRAS, 463, 3582

Mantz A. B. et al., 2015, MNRAS, 446, 2205

McCarthy I. G., Bird S., Schaye J., Harnois-Deraps J., Font

A. S., van Waerbeke L., 2018, MNRAS, 476, 2999

McClintock T. et al., 2019, ApJ, 872, 53

Munari E., Biviano A., Borgani S., Murante G., Fabjan D., 2013,

MNRAS, 430, 2638

Nagai D., 2006, ApJ, 650, 538

Nagai D., Kravtsov A. V., Vikhlinin A., 2007, ApJ, 668, 1

Nagai D., Lau E. T., 2011, ApJ, 731, L10

Nelson K., Lau E. T., Nagai D., 2014, ApJ, 792, 25

Okabe N., Zhang Y. Y., Finoguenov A., Takada M., Smith G. P.,

Umetsu K., Futamase T., 2010, ApJ, 721, 875

Planck Collaboration et al., 2016, A\&A, 594, A24

Planck Collaboration et al., 2017, A\&A, 607, A95

Pratt G. W., Croston J. H., Arnaud M., Böhringer H., 2009, A\&A, 498, 361

Ragagnin A., Dolag K., Moscardini L., Biviano A., D'Onofrio M., 2019, MNRAS, 486, 4001

Rasia E. et al., 2014, ApJ, 791, 96
Rasia E. et al., 2012, New Journal of Physics, 14, 055018

Remus R.-S., Dolag K., Hoffmann T., 2017, Galaxies, 5, 49

Saro A., Mohr J. J., Bazin G., Dolag K., 2013, ApJ, 772, 47

Shi X., Komatsu E., Nagai D., Lau E. T., 2016, MNRAS, 455, 2936

Sifón C. et al., 2013, ApJ, 772, 25

Simet M., McClintock T., Mandelbaum R., Rozo E., Rykoff E., Sheldon E., Wechsler R. H., 2017, MNRAS, 466, 3103

Spiegelhalter D. J., Best N. G., Bradley P. C., van der Linde A., 2002, J. Roy. Stat. Soc., 64, 583

Springel V., 2005, MNRAS, 364, 1105

Springel V., Hernquist L., 2003, MNRAS, 339, 289

Springel V. et al., 2005, Nature, 435, 629

Springel V., White S. D. M., Tormen G., Kauffmann G., 2001, MNRAS, 328, 726

Stafford S. G., McCarthy I. G., Crain R. A., Salcido J., Schaye J., Font A. S., Kwan J., Pfeifer S., 2019, arXiv e-prints, arXiv:1907.09497

Stanek R., Rasia E., Evrard A. E., Pearce F., Gazzola L., 2009, ArXiv e-prints

Sunyaev R. A., Zel'dovich Y. B., 1972, Comments on Astrophysics and Space Physics, 4, 173

Tinker J., Kravtsov A. V., Klypin A., Abazajian K., Warren M., Yepes G., Gottlöber S., Holz D. E., 2008, ApJ, 688, 709

Tinker J. L., Robertson B. E., Kravtsov A. V., Klypin A., Warren M. S., Yepes G., Gottlöber S., 2010, ApJ, 724, 878

Tornatore L., Borgani S., Dolag K., Matteucci F., 2007, MNRAS, 382, 1050

Truong N. et al., 2018, MNRAS, 474, 4089

Velliscig M., van Daalen M. P., Schaye J., McCarthy I. G., Cacciato M., Le Brun A. i. M. C., Dalla Vecchia C., 2014, MNRAS, 442, 2641

Vikhlinin A. et al., 2009, ApJ, 692, 1060

Villaescusa-Navarro F. et al., 2019, arXiv e-prints, arXiv:1909.05273

White M., Cohn J. D., Smit R., 2010, MNRAS, 408, 1818

Wiersma R. P. C., Schaye J., Smith B. D., 2009, MNRAS, 393, 99

Wu H.-Y., Evrard A. E., Hahn O., Martizzi D., Teyssier R., Wechsler R. H., 2015, MNRAS, 452, 1982

Zhuravleva I., Churazov E., Kravtsov A., Lau E. T., Nagai D., Sunyaev R., 2013, MNRAS, 428, 3274

\section{Appendix A $L_{\mathrm{bol}}-M_{\mathrm{vir}}$ scaling relations}

Bolometric gas luminosity $L_{\mathrm{bol}}$ is the sum of emissivity of all gas particles within a given overdensity radius. The self-similar evolution predicts $L_{\mathrm{bol}} \propto M_{\mathrm{vir}}^{4 / 3} F(z)^{7 / 3}$. We find a strong deviation from this prediction as well as a more substantial cosmology dependence of this deviation.

In Figure A.1 we show $\alpha, \beta$ and $\sigma$ for the $L_{\mathrm{bol}}-M_{\mathrm{vir}}$ relation (empty triangles). The dark shaded region represents observational uncertainties taken from Bulbul et al. (2019). All three parameters exhibit a large variation as we move from $\mathrm{C} 1$ to $\mathrm{C} 15$. Most of the X-ray luminosity is associated to the central cluster regions, as $L_{\mathrm{bol}}$ is proportional to the density square. For this reason, it is much more strongly affected by relatively small scale physical processes such as cooling and feedback, which are not naturally accounted by the self-similar prediction (Pratt et al. 2009, Bulbul et al. 2019).

To confirm this, we replace the total bolometric luminosity by the core-subtracted luminosity, $L_{\text {bol }}^{c s}$. Observers generally remove the contribution coming from central $15 \%$ of $R_{500 \text { c }}$ to obtain the core-subtracted luminosity. However, we do not have information stored for individual particles. Instead we have observable quantities for six overdensities, $\Delta_{i}=$ 


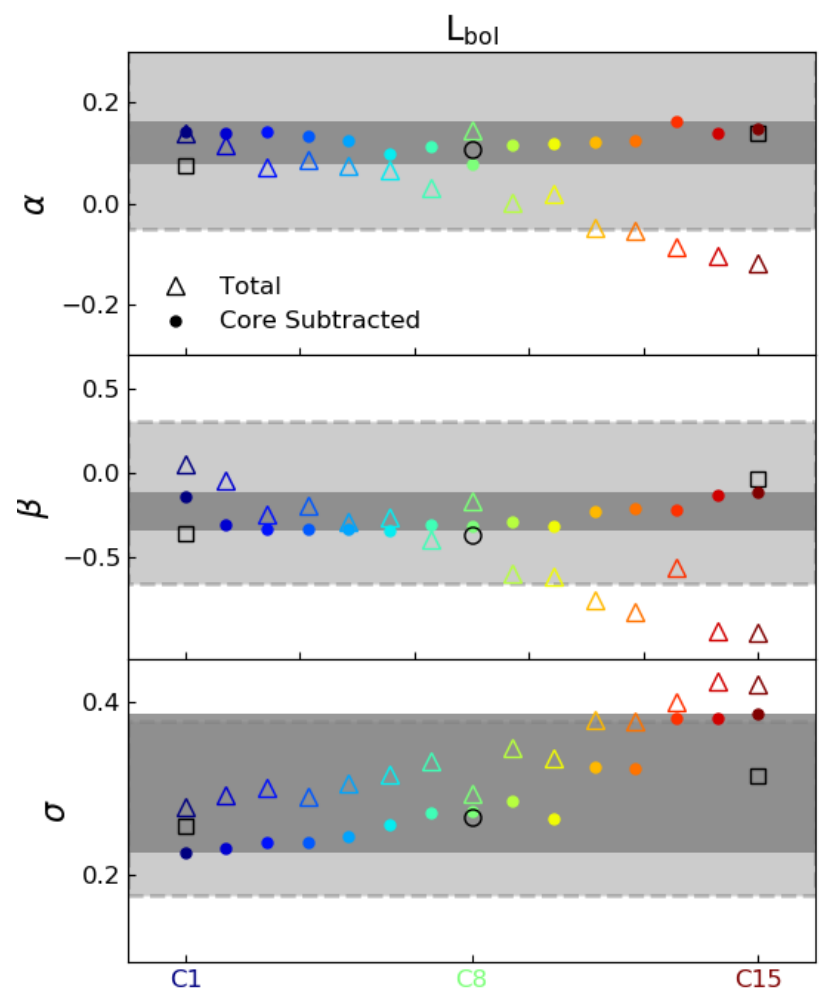

Figure A.1. Empty triangles are for total gas luminosity whereas filled circles are for core-subtracted gas luminosity. Rest is the same as Figure 2

$2500 \mathrm{c}, 500 \mathrm{c}, 500 \mathrm{~m}, 200 \mathrm{c}, 200 \mathrm{~m}$ and vir, where the subscript $\mathrm{c}$ and $\mathrm{m}$ correspond to the critical density and mean matter density of the Universe, respectively. We obtain $L_{\mathrm{bol}}^{c s}$ by removing the contribution coming from the region within $2500 \rho_{c}$, closest the traditional definition of core-subtracted luminosity. This removes most of the the cosmology dependence for the resulting mass and redshift slopes $\alpha$ and $\beta$ (shown as filled circles and the systematic uncertainties shown by light grey shaded region enclosed between dashed lines in Figure A.1. We find that the associated log-normal scatter for the $L_{\mathrm{bol}}^{c s}-M_{\mathrm{vir}}$ relation is reduced, however, it still shows some variation with cosmology.

Results of the fitting procedure for the core-subtracted luminosity are shown in Table A1 There is a minor deviation from the self-similar mass slope compared to the observed core-subtracted $L_{\mathrm{X}, \text { bol }}-M_{500 c}$ relation (Bulbul et al. 2019). Note that the observations are made at a different overdensity and a different definition of core radius. The redshift slope is in agreement with observed as well as the self-similar prediction. The scatter in the scaling relation is $\sim 31 \%$, in a good agreement with the observed scatter in $L_{\mathrm{X}, \mathrm{bol}}-M_{500 c}$ relation. There are large uncertainties on $\gamma_{h_{0}}$ and $\gamma_{\sigma_{8}}$ and both of them consistent with zero within $2-\sigma$. However, $L_{\mathrm{bol}}^{c s}-M_{\mathrm{vir}}$ exhibit a strong dependence on the baryon fraction with $L_{\mathrm{bol}}^{c s} \propto f_{b}^{1.93}$. This dependence is expected as the gas luminosity is strongly depends on the underlying gas density $\left(L_{\mathrm{bol}} \propto \rho^{2}\right)$.
Table A1. Same as Table $4 \& 5$ for $L_{\mathrm{bol}}-M_{\mathrm{vir}}$ scaling relation (core-subtracted bolometric luminosity as defined in Section A).

\begin{tabular}{cc} 
& $L_{\mathrm{bol}}$ \\
\hline$\Pi_{c, 0}$ & $-0.43 \pm 0.52$ \\
$\alpha$ & $0.12 \pm 0.04$ \\
$\beta$ & $-0.22 \pm 0.115$ \\
$\sigma$ & $0.31 \pm 0.08$ \\
\hline & \\
$\gamma_{h_{0}}$ & $1.03 \pm 0.61$ \\
$\gamma_{b}$ & $1.93 \pm 0.06$ \\
$\gamma_{\sigma 8}$ & $-0.37 \pm 0.30$ \\
\hline
\end{tabular}

\section{Appendix B Scaling relations for $M_{500 c}$}

We presented our results for $O-M_{\text {vir }}$ relations in the main analysis. In this section, we present our results for $O-M_{500 \mathrm{c}}$ relations. Again note that the observables are measured within $R_{\Delta_{i}}$ when considering the scaling relation $O-M_{\Delta_{\mathrm{i}}}$ (except for $\sigma_{v}$ which is independent of the over-density definition). Another difference between $M_{\text {vir }}$ and $M_{500 c}$ MORs is the self-similar redshift evolution. We replace $F(z)$ by $E(z)$ in Equation 1 while dealing with $O-M_{500 c}$ relations. The mass and redshift pivots and the redshift cutoff remain the same. The lower mass cutoff is now at $M_{500 c}^{\text {cutoff }}=2 \times 10^{14}$.

In Figure B.1 we show the variation in astrophysical MOR parameters as a function of cosmology and in Table B1 we list the best-fitting results and systematic uncertainties for all six observables. In general, there is a small increase in the variation of

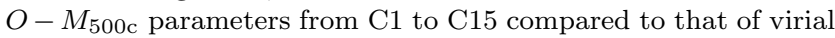
over-density. For $M_{\text {gas }}, M_{\mathrm{b}}, T_{\text {gas }}, \sigma_{v}$ and $Y$, the systematic uncertainties are well within the observational uncertainties. In the case of $L_{\mathrm{bol}}$ and $M_{*}$, systematic uncertainties in the log-normal scatter are comparable to the observed ones, similar to what we found for $M_{\mathrm{vir}}$ scaling relations. Given the systematic uncertainties, there are no major differences between the best-fitting results for $M_{\mathrm{vir}}$ and $M_{500 c}$ (except in the case of the redshift evolution of $\left.L_{\mathrm{bol}}\right)$.

\section{Appendix C Variation in subgrid prescription}

One of the major source of uncertainty in our analysis is fueled by the unknown cosmology dependence of subgrid prescription. In the main analysis, the subgrid model parameters are tuned to the observations at $\mathrm{C} 8$, and we then do not vary these parameters for other cosmologies. In this section, we discuss the impact of variation in subgrid prescription on the MOR parameters.

We run non-radiative versions of $\mathrm{C} 1$ and $\mathrm{C} 15$ cosmologies (shown by empty squares in Figures 2 3.1 \& B.1). To explore the influence of the sub-grid models we modified the most influential parameters defining the strength of the feedback, namely the velocity of the galactic wind (kinetic feedback) as well as the overall strength of the AGN feedback in simulations. For the later we run two simulations where the feedback efficiency of the AGN model was changed from the default value of 0.15 (as used in the simulations varying the cosmology) to either be 0.1 (a1 run) or 0.2 (a2 run). Furthermore, we also explored the influence of the galactic wind feedback, by changing our default value of $350 \mathrm{~km} / \mathrm{s}$ 


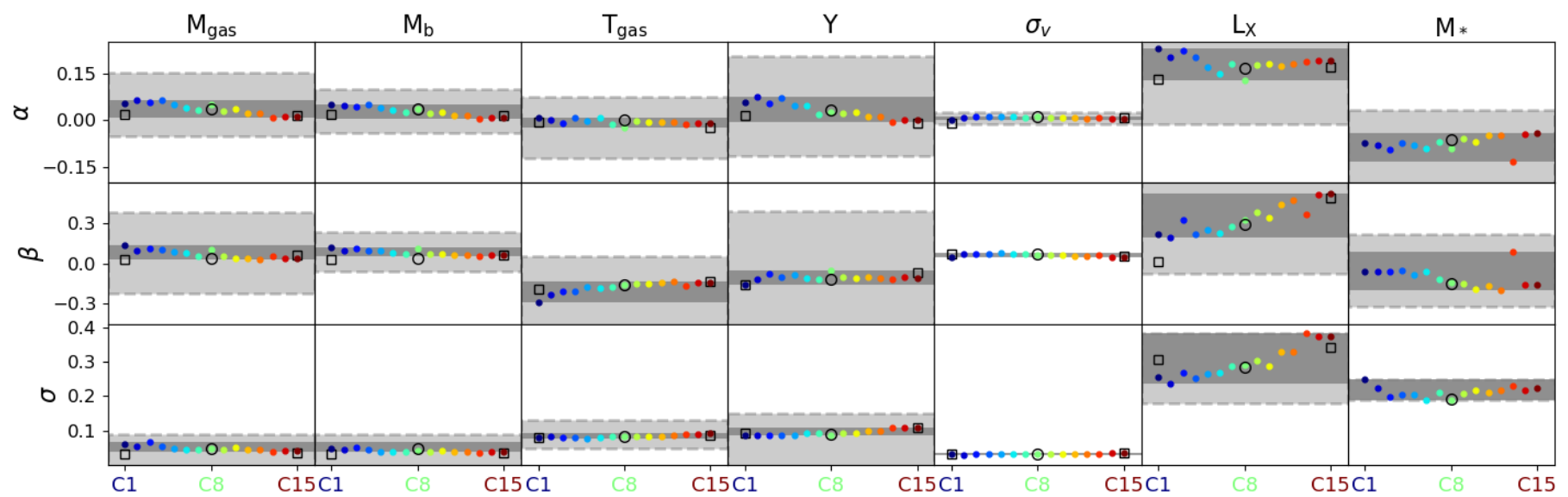

Figure B.1. Same as Figure 2 for $M_{500 c}$ MORs. For gas luminosity, we show only the results for core-subtracted luminosity and for stellar mass, we show the results for $t_{*}(z)$ redshift parametrization with scatter as a function of log-normalization of the scaling relation.

Table B1. Same as Table 4 and A1 for $O-M_{500 c}$ scaling relation.

\begin{tabular}{cccccccc} 
& $M_{\text {gas }}$ & $M_{\mathrm{b}}$ & $M_{*}$ & $T_{\text {gas }}$ & $Y$ & $\sigma_{v}$ & $L_{\mathrm{bol}}$ \\
\hline$\Pi_{c, 0}$ & $31.28 \pm 0.34$ & $31.36 \pm 0.38$ & $28.47 \pm 1.19$ & $1.06 \pm 0.08$ & $32.33 \pm 0.35$ & $6.55 \pm 0.02$ & $0.13 \pm 0.75$ \\
$\alpha$ & $0.04 \pm 0.03$ & $0.03 \pm 0.02$ & $-0.09 \pm 0.04$ & $-0.01 \pm 0.02$ & $0.03 \pm 0.04$ & $0.01 \pm 0.01$ & $0.18 \pm 0.05$ \\
$\beta$ & $0.08 \pm 0.05$ & $0.09 \pm 0.03$ & $-0.05 \pm 0.14$ & $-0.22 \pm 0.08$ & $-0.11 \pm 0.05$ & $0.06 \pm 0.01$ & $0.36 \pm 0.17$ \\
$\sigma$ & $0.05 \pm 0.01$ & $0.04 \pm 0.01$ & $0.22 \pm 0.03$ & $0.08 \pm 0.01$ & $0.10 \pm 0.01$ & $0.032 \pm 0.002$ & $0.31 \pm 0.07$ \\
\hline & & & & & & \\
$\gamma_{h_{0}}$ & $-0.27 \pm 0.27$ & $-0.03 \pm 0.22$ & $1.27 \pm 0.67$ & $0.81 \pm 0.18$ & $0.59 \pm 0.35$ & $0.36 \pm 0.01$ & $1.45 \pm 0.52$ \\
$\gamma_{b}$ & $0.70 \pm 0.04$ & $0.82 \pm 0.04$ & $2.57 \pm 0.06$ & $0.13 \pm 0.05$ & $0.79 \pm 0.06$ & $-0.015 \pm 0.001$ & $1.85 \pm 0.07$ \\
$\gamma_{\sigma_{8}}$ & $-0.22 \pm 0.08$ & $-0.14 \pm 0.06$ & $1.65 \pm 0.39$ & $0.10 \pm 0.05$ & $-0.10 \pm 0.07$ & $-0.05 \pm 0.01$ & $-0.65 \pm 0.24$ \\
\hline
\end{tabular}

to $500 \mathrm{~km} / \mathrm{s}$ (w1 run) and $800 \mathrm{~km} / \mathrm{s}$ (w2 run). While the comparison with the non radiative runs give a general feeling of the importance of the detailed star-formation and black hole model, this should capture the range of reasonable choices of the details within the according sub-grid models. We do not find any significant difference in the best-fitting values of the astrophysical parameters when we vary the subgrid model parameters as shown by empty circles in Figures 2,3, A.1 \& B.1 For clarity, we plot the results for a 2 run only in the figures since the differences between the parameter constraints obtained from different feedback runs are negligible. For most of the observables, the variation is well within the systematic uncertainties and for all of them it is much smaller than the observed uncertainties. 